
others to do so, for U.S. Government purposes. Los Alamos National Laboratory requests that the publisher identify this article others to do so, for U.S. Government purposes. Los Alamos Nark performed under the auspices of the U.S. Department of Energy. The Los Alamos National Laboratory strongly supports academic freedom and a researcher's right to publish; as an institution, however, the Laboratory does not endorse the viewpoint of a publication or guarantee its technical correctness. 


\section{DISCLAMMER}

This report was prepared as an account of work sponsored by an agency of the United States Goverament. Neither the United Stales Governmeat dor any agency thereof, nor any of their employees, makes any warranty, express or implied, or assumes any legal liability or responsibility for the accuracy, completeress, of usefulness of any information, apparatus, product, or process disclosed or represents that its use would not infringe privately owned rights. Refereace beres to any speciilc commercial product, process, or service by trade name. indemart manufac. turer, or otherwise does not necessarily constitute or imply is endorsement. recommendation, or favoring by the United States Government or any ageacy bereof. The views and opinions of authors expressed herein do not oecsuanily sate or reflect those of the United States Government or any agency tbereof. 


\section{DISCLAIMER}

Portions of this document may be illegible in electronic image products. Images are produced from the best available original document. 
Title: Feedback linearization application for LLRF control system

\author{
Authors: Sung-il Kwon, Amy Regan, Y. M. Wang, and T. Rohlev \\ RF Technology Group \\ Accelerator Operations and Technology Division \\ Los Alamos National Laboratory \\ E-mail: skwon@lanl.gov
}

Submitted to: 1999 American Control Conference, San Diego, CA.

Remark: The second sections of the paper entitled "Adaptive feedforward ..." , the paper entitled "Phase synchronization ...", and the paper entitled "Feedback linearization ..." are describing the klystron model and are almost same. Also, the third sections of the paper entitled "Adaptive feedforward ..." and the paper entitled "Feedback linearization ..." are describing the RF cavity model and almost same. The rest sections of each paper describe the different control techniques and they are derived from the klystron model and the RF cavity model described in the second section and the third section. When at least two papers are accepted for full papers, the second section and the third section will be modified. 


\title{
Feedback linearization Application for LLRF Control System
}

\author{
Sung-il Kwon, Amy Regan, Y. M. Wang, and T. Rohlev \\ RF Technology Group \\ Accelerator Operations and Technology Division \\ Los Alamos National Laboratory \\ P.O.Box 1663 \\ Los Alamos, NM 87544, USA \\ E-mail: skwon@lanl.gov
}

\begin{abstract}
The Low Energy Demonstration Accelerator(LEDA) being constructed at Los Alamos National Laboratory will serve as the prototype for the low energy section of Acceleration Production of Tritium(APT) accelerator. This paper addresses the problem of the LLRF control system for LEDA. We propose a control law which is based on exact feedback linearization coupled with gain scheduling which reduces the effect of the deterministic klystron cathode voltage ripple that is due to harmonics of the high voltage power supply and achieves tracking of desired set points. Also, we propose an estimator of the ripple and its time derivative and the estimates based feedback linearization controller.
\end{abstract}

\section{Introduction}

The low energy demonstration accelerator(LEDA) for the Production of Tritium(APT) is being built at Los Alamos National Laboratory. The primary function of the low level RF(LLRF) control system of LEDA is to control RF fields in the accelerating Cavity and maintain field stability within $\pm 1 \%$ peak to peak amplitude error and $1^{\circ}$ peak to peak phase error[8]. 
This paper addresses the problem of the LLRF control system used for LEDA. We propose a control law which is based on exact feedback linearization[3] coupled with gain scheduling[9],[10]. The purpose of exact feedback linearization coupled with gain scheduling is to reduce the effect of the deterministic cathode ripple that is due to harmonics of high voltage power supply[7] and is to achieve tracking of desired set points. Low frequency ripple does not deteriorate the current LLRF control system based on PID control method. As frequency of ripple increases, the effect of the ripple on the performance increases too. Simulation shows that $0.3 \%$ high voltage power supply ripple yields $1.05^{\circ}$ at about $72 \mathrm{kHz}$ [5] and $1.0 \%$ high voltage power supply ripple yields $3.6^{\circ}$ at about $120 \mathrm{kHz}$ [12]. In order to suppress the high frequency ripple, the proposed controller makes use of not only the ripple but also the time derivative of the ripple. The usage of time derivative of the ripple improve the controller performance[10]. First, we assume that the deterministic cathode ripple is measurable and derive the controller. Second, we propose the ripple estimator which estimates the ripple signal itself and the time derivative of the ripple as well and derive the controller coupled with the ripple estimator. As is well known, in order to design the exact feedback linearization controller, the given system to be controlled must be well defined. Previous works[11], [13] modeled the klystron and RF Cavity used for LEDA. Our current work is based on the klystron model and RF Cavity model set up in Matlab/Simulink environment.

\section{The Klystron Model}

We consider a klystron model as shown in Figure 1.

It has two inputs, LLRF_I and LLRF_Q and two output HPRF_I and HPRF_Q. As intermediate outputs, Klystron has the normalized amplitude N_AMPLITUDE and the normalized phase N_PHASE.

The first stage of a klystron are linear systems called FILTER AND AMPLIFIER. Let $u_{1}=$ LLRF_I and let $u_{2}=$ LLRF_Q . Let $x_{1}$ and $x_{2}$ be outputs of the systems whose transfer function are given by

$$
\begin{aligned}
& \frac{X_{1}(s)}{U_{1}(s)}=\frac{1}{3.54 e^{-7} s+1} \\
& \frac{X_{2}(s)}{U_{2}(s)}=\frac{1}{3.54 e^{-7} s+1} .
\end{aligned}
$$

In state space, transfer functions (1) and (2) are represented as

$$
\dot{x}_{1}=-a_{1} x_{1}+a_{1} u_{1}
$$




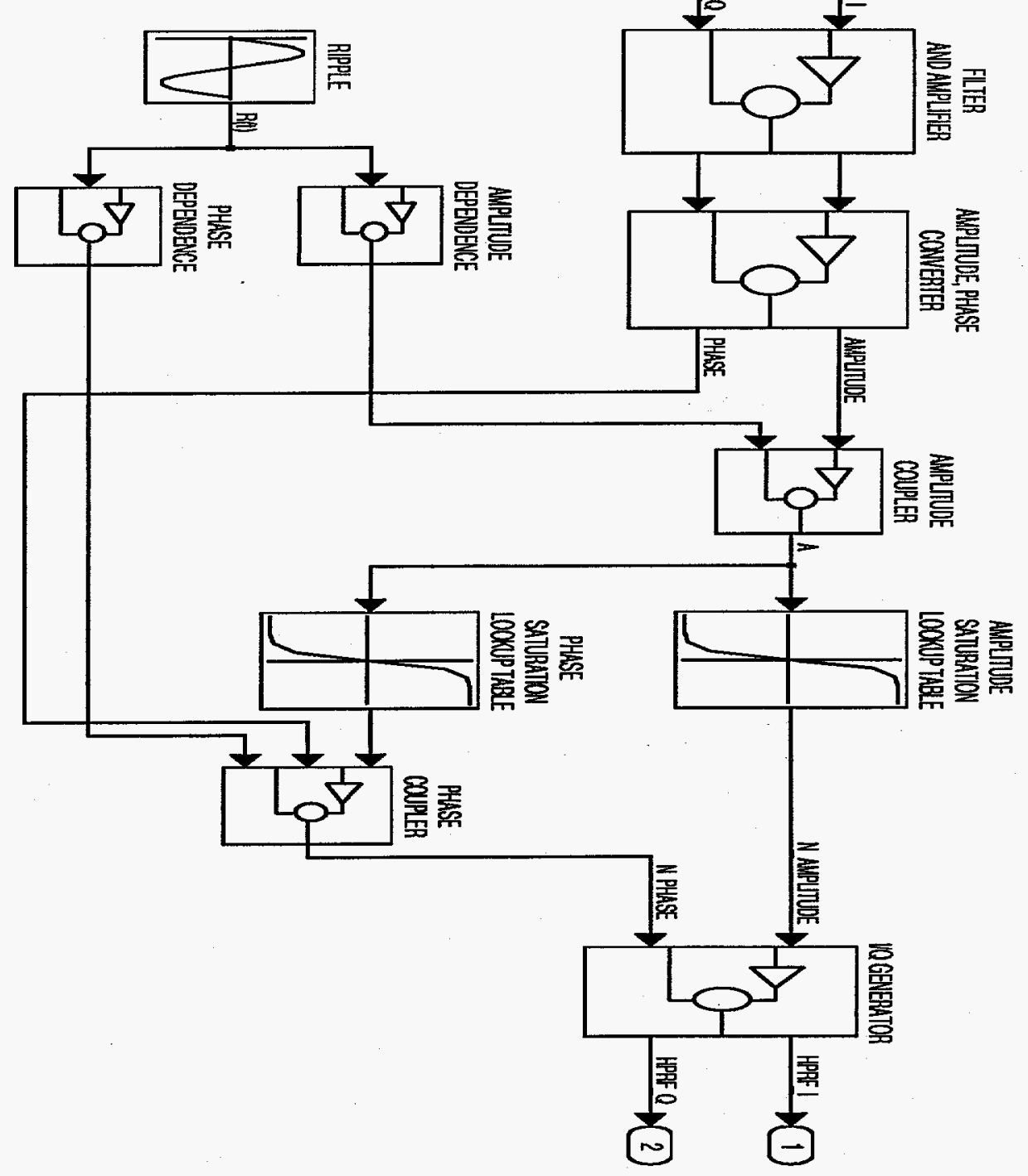

Figure 1: A Klystron Model 


$$
\dot{x}_{2}=-a_{1} x_{2}+a_{1} u_{2}
$$

where $a_{1}=\frac{1 e^{+007}}{3.54}$.

A klystron model has two loop-up tables, called AMPLITUDE SATURATION and PHASE SATURATION. The input of the two look-up tables is given by

$$
A=\frac{K_{g}}{10 \sqrt{K P_{m}}}(0.01 R(t)+1)^{1.25} \cdot \sqrt{x_{1}^{2}+x_{2}^{2}}
$$

where $R(t)$ is the ripple, $K_{g}$ is the klystron gain, and $K P_{m}$ is the maximum klystron power. $R(t), K_{g}$, and $K P_{m}$ are specified for a given klystron. For given $\mathrm{A}$, the output of the look-up table AMPLITUDE SATURATION can be represented by

$$
A_{N}=I_{1}(A)
$$

and the output of the look-up table PHASE SATURATION can be represented by

$$
\theta_{N}=I_{2}(A)
$$

Table 1 and table 2 show data of look-up table AMPLITUDE SATURATION and data of look-up table PHASE SATURATION, respectively.

\begin{tabular}{||r|r||r|r||r|r||r|r||}
\hline \hline$A$ & $A_{N}$ & $A$ & $A_{N}$ & $A$ & $A_{N}$ & $A$ & $A_{N}$ \\
\hline \hline-0.1000 & 0.0000 & 0.0700 & 0.0000 & 0.1400 & 0.1900 & 0.5700 & 0.7500 \\
\hline 0.7100 & 0.8700 & 0.8600 & 0.9800 & 0.9000 & 1.0000 & 0.9100 & 1.0000 \\
\hline 0.3122 & 0.4143 & 0.3568 & 0.4724 & 0.4014 & 0.5305 & 0.4461 & 0.5886 \\
\hline 0.4907 & 0.6467 & 0.5353 & 0.7048 & 0.5799 & 0.7585 & 0.5910 & 0.7680 \\
\hline 1.0000 & 0.9900 & 0.4461 & 0.5886 & 0.6468 & 0.8158 & 0.6691 & 0.8349 \\
\hline 0.0446 & 0.0000 & 0.0892 & 0.0521 & 0.6914 & 0.8540 & 0.7360 & 0.8891 \\
\hline 0.1338 & 0.1732 & 0.1784 & 0.2400 & 0.7806 & 0.9218 & 0.8252 & 0.9545 \\
\hline 0.2230 & 0.2981 & 0.2676 & 0.3562 & 0.8921 & 0.9961 & 0.9367 & 0.9970 \\
\hline 0.3122 & 0.4143 & 0.3568 & 0.4724 & 0.9813 & 0.9921 & & \\
\hline \hline
\end{tabular}

Table 1. AMPLITUDE SATURATION Data 


\begin{tabular}{||r|r||r|r||r|r||r|r||}
\hline \hline$A$ & $\theta_{N}$ & $A$ & $\theta_{N}$ & $A$ & $\theta_{N}$ & $A$ & $\theta_{N}$ \\
\hline \hline-0.1000 & 0.0000 & 0.0700 & 0.0000 & 0.6400 & -0.0150 & 0.7100 & -0.0350 \\
\hline 0.8600 & -0.1370 & 0.9000 & -0.2440 & 1.0000 & -0.4770 & 0.0446 & 0.0000 \\
\hline 0.4987 & -0.0113 & 0.5445 & -0.0125 & 0.5576 & -0.0128 & 0.6691 & -0.0233 \\
\hline 0.0892 & $-5.0552 \mathrm{e}-4$ & 0.1338 & -0.0017 & 0.5712 & -0.0132 & 0.7140 & -0.0377 \\
\hline 0.1784 & -0.0029 & 0.2230 & -0.0040 & 0.8921 & -0.2229 & 0.4549 & -0.0101 \\
\hline 0.2676 & -0.0052 & 0.3122 & -0.0064 & 0.4483 & -0.0100 & 0.4014 & -0.0087 \\
\hline 0.3568 & -0.0075 & 0.7885 & -0.0884 & 0.9593 & -0.3821 & & \\
\hline \hline
\end{tabular}

Table 2. PHASE SATURATION Data

The normalized amplitude N_Amplitude, defined by $y_{1}^{k}$ and the normalized phase N_Phase, defined by $y_{2}^{k}$ of the klystron are expressed by

$$
\begin{aligned}
y_{1}^{k} & =A_{N}=I_{1}(A) \\
y_{2}^{k} & =\theta_{N}+\tan ^{-1}\left(\frac{x_{2}}{x_{1}}\right)+3 \cdot \frac{\pi}{180} \cdot R(t) \\
& =I_{2}(A)+\tan ^{-1}\left(\frac{x_{2}}{x_{1}}\right)+3 \cdot \frac{\pi}{180} \cdot R(t) .
\end{aligned}
$$

In addition, for given $y_{1}$ and $y_{2}$, HPRF_I and HPRF_Q are given by

$$
\begin{aligned}
& H P R F_{-} I=10 \sqrt{K P_{m}} \cdot y_{1}^{k} \cdot \cos \left(y_{2}^{k}\right) \\
& H P R F_{-} Q=10 \sqrt{K P_{m}} \cdot y_{1}^{k} \cdot \sin \left(y_{2}^{k}\right) .
\end{aligned}
$$

Since the look-up tables have the limited number of data, we need to approximate the look-up tables by linear or nonlinear curve fitting equations. Considering the characteristic curve of a klystron, we choose nonlinear equations. We choose curve fitting equations of AMPLITUDE SATURATION and PHASE SATURATION having the forms

$$
\begin{aligned}
& A_{N}=\sum_{i=1}^{N} c_{i} e^{-f_{i} A} \\
& \theta_{N}=\sum_{i=1}^{N} d_{i} e^{-f_{i} A}
\end{aligned}
$$


where $f_{i}, i=1,2, \cdots, N$ and parameters $c_{i}, i=1,2, \cdots, N, d_{i}, i=1,2, \cdots, N$ are to be determined.

Higher order of a curve fitting equation may yield more accurate curve fitting equation. For simplicity, we choose $N=7$. Also, in order to reduce the number of coefficients to be determined, $f_{i}, i=1,2, \cdots, N$ are given in Table 3 .

\begin{tabular}{|r|r|r|r|r|r|r||}
\hline \hline$f_{1}$ & $f_{2}$ & $f_{3}$ & $f_{4}$ & $f_{5}$ & $f_{6}$ & $f_{7}$ \\
\hline \hline 0.50 & 0.75 & 1.00 & 1.25 & 1.50 & 1.75 & 2.00 \\
\hline \hline
\end{tabular}

Table 3. Exponents of curve fitting equations

By using data given in Table 1 and Table 2, we obtain coefficients $c_{i}, i=1,2, \cdots, N$ and $d_{i}, i=1,2, \cdots, N$, of the curve fitting equations (12) and (13). Coefficients $c_{i}$ and $d_{i}$ obtained are given in Table 4 . Figure 2 shows plots of data points as given in Table 4, Table 5 and plots of curve fitting equations (12) and (13) whose coefficients, $f_{i}, i=1,2, \cdots, N$, $c_{i}, i=1,2, \cdots, N, d_{i}, i=1,2, \cdots, N$, are given in Table 3 and Table 4 with appropriate domain of $A$.

\begin{tabular}{||l|r||c|r||}
\hline \hline$c_{1}$ & $0.05680429876058 \mathrm{e}+006$ & $d_{1}$ & $-0.14120739315590 \mathrm{e}+005$ \\
\hline$c_{2}$ & $-0.39264357353961 \mathrm{e}+006$ & $d_{2}$ & $0.83084262097993 \mathrm{e}+005$ \\
\hline$c_{3}$ & $1.12805594234952 \mathrm{e}+006$ & $d_{3}$ & $-2.01778226478032 \mathrm{e}+005$ \\
\hline$c_{4}$ & $-1.72418545240933 \mathrm{e}+006$ & $d_{4}$ & $2.58441412755651 \mathrm{e}+005$ \\
\hline$c_{5}$ & $1.47878241712872 \mathrm{e}+006$ & $d_{5}$ & $-1.83680595711727 \mathrm{e}+005$ \\
\hline$c_{6}$ & $-0.67483667002473 \mathrm{e}+006$ & $d_{6}$ & $0.68453128529433 \mathrm{e}+005$ \\
\hline$c_{7}$ & $0.12802296547207 \mathrm{e}+006$ & $d_{7}$ & $-0.10399245992504 \mathrm{e}+005$ \\
\hline
\end{tabular}

Table 4. Coefficients of curve fitting equations

Plugging (5) to (12) and (13), curve fitting equations (12) and (13) are reduced to

$$
\begin{aligned}
& A_{N}=\sum_{i=1}^{N} c_{i} e^{-f_{i} w(t) \sqrt{x_{1}^{2}+x_{2}^{2}}} \\
& \theta_{N}=\sum_{i=1}^{N} d_{i} e^{-f_{i} w(t) \sqrt{x_{1}^{2}+x_{2}^{2}}}
\end{aligned}
$$


where

$$
w(t)=\frac{K_{g}}{10 \sqrt{K P_{m}}}(0.01 R(t)+1)^{1.25} .
$$

The normalized amplitude $y_{1}^{k}$ and the normalized phase $y_{2}^{k}$ of the klystron are

$$
\begin{aligned}
y_{1}^{k} & =A_{N}=\sum_{i=1}^{N} c_{i} e^{-f_{i} w(t) \sqrt{x_{1}^{2}+x_{2}^{2}}} \\
y_{2}^{k} & =\theta_{N}+\tan ^{-1}\left(\frac{x_{2}}{x_{1}}\right)+3 \cdot \frac{\pi}{180} \cdot R(t) \\
& =\sum_{i=1}^{N} d_{i} e^{-f_{i} w(t) \sqrt{x_{1}^{2}+x_{2}^{2}}}+\tan ^{-1}\left(\frac{x_{2}}{x_{1}}\right)+3 \cdot \frac{\pi}{180} \cdot R(t) .
\end{aligned}
$$

In addition, for given $y_{1}^{k}$ and $y_{2}^{k}$, HPRF_I and HPRF_Q are given by

$$
\begin{aligned}
& H P R_{-} I=10 \sqrt{K P_{m}} \cdot y_{1}^{k} \cdot \cos \left(y_{2}^{k}\right) \\
& H P R_{-} Q=10 \sqrt{K P_{m}} \cdot y_{1}^{k} \cdot \sin \left(y_{2}^{k}\right) .
\end{aligned}
$$

\subsection{The Klystron in $z$-coordinate}

Consider the normalized amplitude $y_{1}^{k}$ and the normalized phase $y_{2}^{k}$ as given in (17) and (18).

Let

$$
\begin{aligned}
& z_{1}=\sqrt{x_{1}^{2}+x_{2}^{2}} \\
& z_{2}=\tan ^{-1}\left(\frac{x_{2}}{x_{1}}\right) .
\end{aligned}
$$

We consider a transformation from $x$-coordinate to $z$-coordinate. In $z$-coordinate, the state equations (3) and (4) are reduced to

$$
\begin{aligned}
& \dot{z}_{1}=-a_{1} z_{1}+a_{1} \cos \left(z_{2}\right) u_{1}+a_{1} \sin \left(z_{2}\right) u_{2} \\
& \dot{z}_{2}=-a_{1} \frac{\sin \left(z_{2}\right)}{z_{1}} u_{1}+a_{1} \frac{\cos \left(z_{2}\right)}{z_{1}} u_{2} .
\end{aligned}
$$

Also, the curve fitting equations (12) and (13) are reduced to

$$
\begin{aligned}
& A_{N}=\sum_{i=1}^{N} c_{i} e^{-f_{i} w(t) z_{1}} \\
& \theta_{N}=\sum_{i=1}^{N} d_{i} e^{-f_{i} w(t) z_{1}} .
\end{aligned}
$$



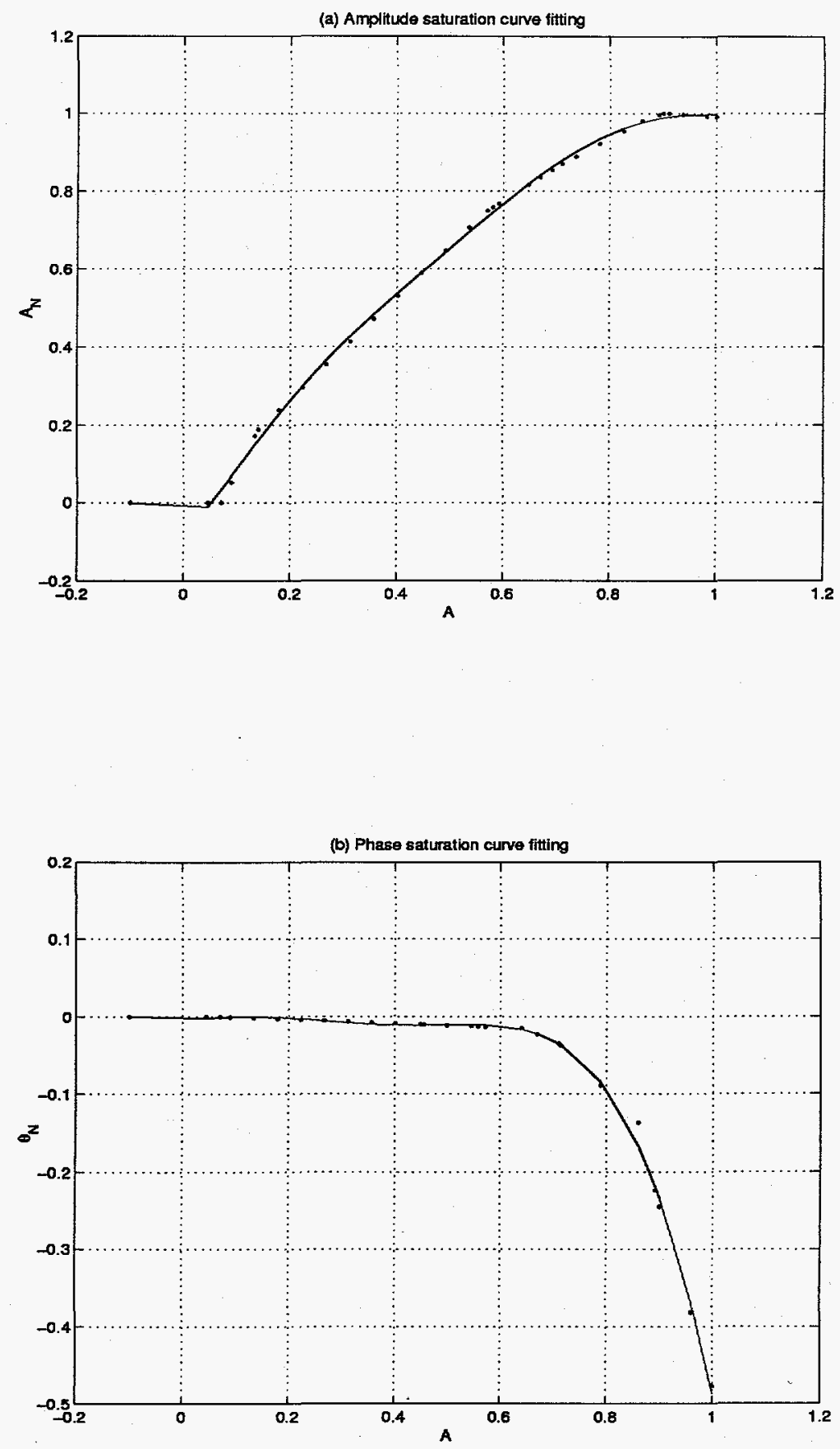

Figure 2: Curve fittings 
The normalized amplitude $y_{1}^{k}$ and the normalized phase $y_{2}^{k}$ are represented by

$$
\begin{aligned}
& y_{1}^{k}=\sum_{i=1}^{N} c_{i} e^{-f_{i} w(t) z_{1}} \\
& y_{2}^{k}=\sum_{i=1}^{N} d_{i} e^{-f_{i} w(t) z_{1}}+z_{2}+3 \cdot \frac{\pi}{180} \cdot R(t) .
\end{aligned}
$$

Note that the exponents of the first term of (27) are the same as the exponents of the first term of (28). Also, note that the phase $y_{2}^{k}$ is linear with respect to $z_{2}$.

\section{The RF Cavity}

Figure 3 shows the RF cavity.

RF Cavity has has four inputs, HPRF_I, HPRF_Q, BEAM_I, and BEAM_Q and two outputs, CAV_FLD_, CAV_FLD_Q.

Let $u_{1}^{c}=$ HPRF_I, $u_{2}^{c}=$ HPRF_Q,$u_{3}^{c}=$ BEAM_I, $u_{4}^{c}=$ BEAM_Q and let $y_{1}^{c}=$ CAV_FLD_I, $y_{2}^{c}=$ CAV_FLD_ Then RF Cavity can be expressed in the state space form.

$$
\begin{aligned}
& \dot{x}=A x+B u^{c} \\
& y^{c}=C x
\end{aligned}
$$

where

$$
A=\left[\begin{array}{cccccccc}
a-\frac{1}{50} c_{1} & b-\frac{1}{50} c_{2} & 0 & 0 & 0 & 0 & \frac{1}{50} c_{3} & \frac{1}{50} c_{4} \\
1 & 0 & 0 & 0 & 0 & 0 & 0 & 0 \\
-\frac{1}{50} c_{1} & -\frac{1}{50} c_{2} & a & b & 0 & 0 & \frac{1}{50} c_{3} & \frac{1}{50} c_{4} \\
0 & 0 & 1 & 0 & 0 & 0 & 0 & 0 \\
0 & 0 & -\frac{1}{50} c_{3} & -\frac{1}{50} c_{4} & a-\frac{1}{50} c_{1} & b-\frac{1}{50} c_{2} & 0 & 0 \\
0 & 0 & 0 & 0 & 1 & 0 & 0 & 0 \\
0 & 0 & -\frac{1}{50} c_{3} & -\frac{1}{50} c_{4} & -\frac{1}{50} c_{1} & -\frac{1}{50} c_{2} & a & b \\
0 & 0 & 0 & 0 & 0 & 0 & 1 & 0
\end{array}\right],
$$




$$
\begin{aligned}
& B=\left[\begin{array}{cccc}
\frac{2}{50} & 0 & -2 e t a & 0 \\
0 & 0 & 0 & 0 \\
\frac{2}{50} & 0 & -2 e t a & 0 \\
0 & 0 & 0 & 0 \\
0 & \frac{2}{50} & 0 & -2 e t a \\
0 & 0 & 0 & 0 \\
0 & \frac{2}{50} & 0 & -2 e t a \\
0 & 0 & 0 & 0
\end{array}\right] \\
& C=\left[\begin{array}{cccccccc}
c_{1} & c_{2} & 0 & 0 & 0 & 0 & -c_{3} & -c_{4} \\
0 & 0 & c_{3} & c_{4} & c_{1} & c_{2} & 0 & 0
\end{array}\right] \\
& a=-\frac{2}{\tau} \\
& b=-\left(\frac{1}{\tau^{2}}+K D W^{2}\right) \\
& c_{1}=\frac{K R}{\tau} \\
& c_{2}=\frac{K R}{\tau}\left(\frac{1}{\tau}-\frac{K D W}{2 K Q o}\right) \\
& c_{3}=\frac{K R}{2 \tau \cdot K Q o} \\
& c_{4}=\frac{K R}{2 \tau K Q o}\left(\frac{1}{\tau}+2 K D W \cdot K Q o\right) .
\end{aligned}
$$

Parameters of RF Cavity are given in [11].

Also, FLD_I and FLD_Q of the Cavity Field Sample System are given by

$$
\begin{aligned}
& F L D_{-} I=F A \cdot \cos (G D) \cdot y_{1}^{c}-F A \cdot \sin (G D) \cdot y_{2}^{c} \\
& F L D_{-} Q=F A \cdot \sin (G D) \cdot y_{1}^{c}+F A \cdot \cos (G D) \cdot y_{2}^{c}
\end{aligned}
$$

and FLD_AMP and FLD_PHS of the Cavity Field Sample System are given by

where

$$
\begin{aligned}
& F L D \_A M P=\sqrt{F L D I^{2}+F L D_{-} Q^{2}} \\
& F L D \_P H S=\tan ^{-1}\left(\frac{F L D_{-} Q}{F L D_{-} I}\right)
\end{aligned}
$$

$$
\begin{aligned}
& F A=0.00037809 \\
& G D=\frac{\pi}{180} \cdot(-0.039455) .
\end{aligned}
$$

The RF Cavity as given in (29), (30) is Hurwitz stable and is inverse stable as well. 


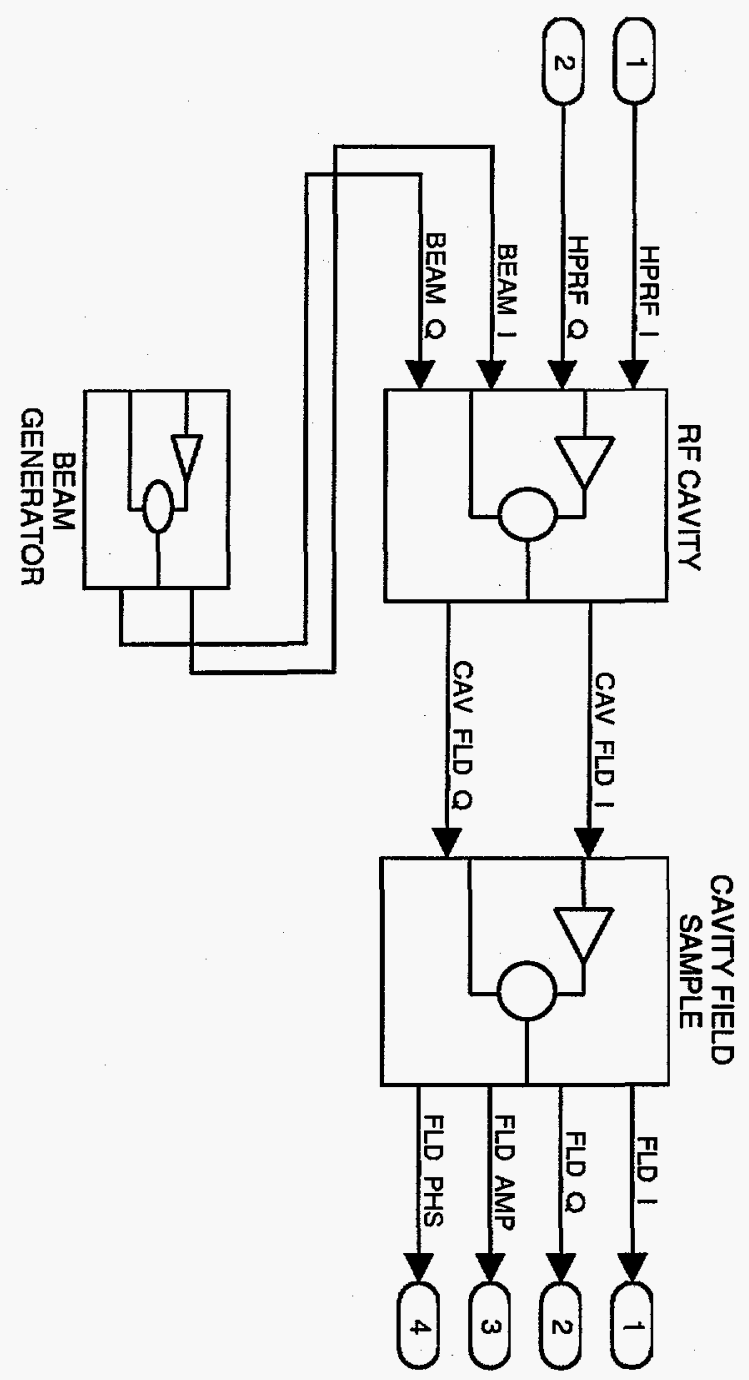

Figure 3: A RF Cavity Model and a Cavity Field Sample System 


\section{The Feedback Linearization Controller}

Consider the Klystron equation in z-coordinate.

$$
\begin{aligned}
& \dot{z}_{1}=-a_{1} z_{1}+a_{1} \cos \left(z_{2}\right) u_{1}+a_{1} \sin \left(z_{2}\right) u_{2} \\
& \dot{z}_{2}=-a_{1} \frac{\sin \left(z_{2}\right)}{z_{1}} u_{1}+a_{1} \frac{\cos \left(z_{2}\right)}{z_{1}} u_{2} .
\end{aligned}
$$

and

$$
\begin{aligned}
& y_{1}^{k}=\sum_{i=1}^{N} c_{i} e^{-f_{i} w(t) z_{1}} \\
& y_{2}^{k}=\sum_{i=1}^{N} d_{i} e^{-f_{i} w(t) z_{1}}+z_{2}+3 \cdot \frac{\pi}{180} \cdot R(t)
\end{aligned}
$$

where

$$
\begin{aligned}
& w(t)=M(0.01 R(t)+1)^{1.25} \\
& M=\frac{K_{g}}{10 \sqrt{K P_{m}}} .
\end{aligned}
$$

Define

$$
\begin{aligned}
& \bar{z}_{1}=(0.01 R(t)+1)^{1.25} z_{1} \\
& \bar{z}_{2}=z_{2}+3 \cdot \frac{\pi}{180} \cdot R(t) .
\end{aligned}
$$

The Klystron is expressed in $\bar{z}$-coordinate by

$$
\begin{aligned}
\dot{\bar{z}}_{1} & =-\bar{a}_{1} \bar{z}_{1}+b_{z 11}(\bar{z}, R(t), \dot{R}(t)) u_{1}+b_{z 12}(\bar{z}, R(t), \dot{R}(t)) u_{2}+E_{z 1}(R(t), \dot{R}(t)) \\
\dot{\bar{z}}_{2} & =b_{z 21}(\bar{z}, R(t), \dot{R}(t)) u_{1}+b_{z 22}(\bar{z}, R(t), \dot{R}(t)) u_{2}+E_{z 2}(R(t), \dot{R}(t)) \\
y_{1}^{k} & =\sum_{i=1}^{N} c_{i} e^{-f_{i} M \bar{z}_{1}} \\
y_{2}^{k} & =\sum_{i=1}^{N} d_{i} e^{-f_{i} M \bar{z}_{1}}+\bar{z}_{2} .
\end{aligned}
$$

where

$$
\begin{aligned}
& \bar{a}_{1}=a_{1}-0.0125(0.01 R(t)+1)^{-1} \dot{R}(t) \\
& b_{z 11}(\bar{z}, R(t), \dot{R}(t))=a_{1}(0.01 R(t)+1)^{1.25} \cos \left(\bar{z}_{2}-3 \cdot \frac{\pi}{180} \cdot R(t)\right)
\end{aligned}
$$




$$
\begin{aligned}
& b_{z 12}(\bar{z}, R(t), \dot{R}(t))=a_{1}(0.01 R(t)+1)^{1.25} \sin \left(\bar{z}_{2}-3 \cdot \frac{\pi}{180} \cdot R(t)\right) \\
& b_{z 21}(\bar{z}, R(t), \dot{R}(t))=-a_{1} \frac{\sin \left(\bar{z}_{2}-3 \cdot \frac{\pi}{180} \cdot R(t)\right)}{\bar{z}_{1}} \\
& b_{z 22}(\bar{z}, R(t), \dot{R}(t))=a_{1} \frac{\cos \left(\bar{z}_{2}-3 \cdot \frac{\pi}{180} \cdot R(t)\right)}{\bar{z}_{1}} \\
& E_{z 1}(R(t), \dot{R}(t))=0, \\
& E_{z 2}(R(t), \dot{R}(t))=3 \cdot \frac{\pi}{180} \dot{R}(t)
\end{aligned}
$$

Note that $\bar{B}_{z}(\bar{z}, R(t), \dot{R}(t))$ is invertible for any nonzero $\bar{z}_{1}$. In $\bar{z}$-coordinate, state equations are dependent upon the ripple $R(t)$ but the output equations are independent upon the ripple $R(t)$.

Define

$$
\begin{gathered}
\bar{A}_{z}(R(t), \dot{R}(t))=\left[\begin{array}{cc}
-\bar{a}_{1} & 0 \\
0 & 0
\end{array}\right], \\
\bar{z}=\left[\begin{array}{c}
\bar{z}_{1} \\
\bar{z}_{2}
\end{array}\right], \quad u_{z}=\left[\begin{array}{l}
u_{1} \\
u_{2}
\end{array}\right], \\
E_{z}(R(t), \dot{R}(t))=\left[\begin{array}{c}
0 \\
3 \cdot \frac{\pi}{180} \dot{R}(t)
\end{array}\right],
\end{gathered}
$$

and

$$
\bar{B}_{z}(\bar{z}, R(t), \dot{R}(t))=\left[\begin{array}{ll}
b_{z 11}(\bar{z}, R(t), \dot{R}(t)) & b_{z 12}(\bar{z}, R(t), \dot{R}(t)) \\
b_{z 21}(\bar{z}, R(t), \dot{R}(t)) & b_{z 22}(\bar{z}, R(t), \dot{R}(t))
\end{array}\right]
$$

Then, (39) and (40) are represented by

$$
\dot{\bar{z}}=\bar{A}_{z}(R(t), \dot{R}(t)) \bar{z}+\bar{B}_{z}(\bar{z}, R(t), \dot{R}(t)) u_{z}+E_{z}(R(t), \dot{R}(t))
$$

Assume that $\bar{z}_{1} \neq 0$. Define

$$
u_{z}=\bar{B}_{z}^{-1}(\bar{z}, R(t), \dot{R}(t))\left(\bar{u}-E_{z}\right)
$$

Then, (48) is reduced to

$$
\dot{\bar{z}}=\bar{A}_{z}(R(t), \dot{R}(t)) \bar{z}+I_{2 \times 2} \bar{u} .
$$


Let

$$
\begin{aligned}
\bar{u} & =K \bar{z}+R_{z} \\
& =\left[\begin{array}{cc}
k_{1}+0.0125(0.01 R(t)+1)^{-1} \dot{R}(t) & 0 \\
0 & k_{2}
\end{array}\right] \bar{z}+\left[\begin{array}{l}
r_{1} \\
r_{2}
\end{array}\right]
\end{aligned}
$$

where $R_{z}$ is the control input that drives the steady state value $\bar{z}_{s}$ of $\bar{z}$ to the desired value of $\bar{z}[2]$. Then, $(50)$ is reduced to

$$
\dot{\bar{z}}=\bar{A}_{z c} \bar{z}+I_{2 \times 2} R_{z}
$$

where

$$
\bar{A}_{z c}=\left[\begin{array}{cc}
-a_{1}+k_{1} & 0 \\
0 & k_{2}
\end{array}\right]
$$

$\bar{A}_{z c}$ is a constant matrix and is independent of $R(t)$ and $\dot{R}(t)$. By proper choice of $k_{1}$ and $k_{2}$, we can locate the eigenvalues of the matrix $\bar{A}_{z c}$ that what we want.

$R_{z}$ is the solution of the following equation[2].

$$
0=\bar{A}_{z c} \bar{z}_{s}+I_{2 \times 2} R_{z}
$$

The solution of (54) can be explained by the steady state value of the transfer function from $R_{z}$ to $\bar{z}$. The transfer function from $R_{z}$ to $\bar{z}$ is

$$
\frac{\bar{z}(s)}{R_{z}(s)}=\left(s I-\bar{A}_{z c}\right)^{-1} I_{2 \times 2}
$$

For constant $R_{z}$, steady state value $\bar{z}_{s}$ of $\bar{z}$ is given by the equation

$$
\bar{z}_{s}=\left(0 I-\bar{A}_{z c}\right)^{-1} I_{2 \times 2} R_{z}
$$

Equivalently,

$$
0=\bar{A}_{z c} \bar{z}_{s}+R_{z}
$$

The solution $R_{z}$ of (54) is represented by the steady state value $\bar{z}_{s}$ of $\bar{z}$ and it is obtained as follow.

First, we consider the equations for FLD_I and FLD_Q as given in (31) and (32).

$$
\left[\begin{array}{c}
F L D I \\
F L D_{-} Q
\end{array}\right]=\left[\begin{array}{cc}
F A \cdot \cos (G D) & -F A \cdot \sin (G D) \\
F A \cdot \sin (G D) & F A \cdot \cos (G D)
\end{array}\right]\left[\begin{array}{l}
y_{1}^{c} \\
y_{2}^{c}
\end{array}\right] \text {. }
$$


Let FLD $I_{d}$ and FLD_Q ${ }_{d}$ be the desired values of FLD_I and FLD_Q. Then, the desired values $y_{1 d}^{c}, y_{2 d}^{c}$ of $y_{1}^{c}, y_{2}^{c}$ are given by the algebraic equation

$$
\left[\begin{array}{l}
y_{1 d}^{c} \\
y_{2 d}^{c}
\end{array}\right]=\left[\begin{array}{cc}
F A \cdot \cos (G D) & -F A \cdot \sin (G D) \\
F A \cdot \sin (G D) & F A \cdot \cos (G D)
\end{array}\right]^{-1}\left[\begin{array}{c}
F L D I_{d} \\
F L D Q_{d}
\end{array}\right]
$$

Second, we consider the Cavity equation.

$$
\begin{aligned}
& \dot{x}=A x+B u^{c} \\
& y^{c}=C x
\end{aligned}
$$

Let $H_{C A V}(s)$ be the transfer function from $u_{1}^{c}=$ HPRF_I and $u_{2}^{c}=$ HPRF_Q to $y_{1}^{c}=$ CAV_FLD_I and $y_{2}^{c}=$ CAV_FLD_Q, assuming that $u_{3}^{c}=$ BEAM_I and $u_{4}^{c}=$ BEAM_Q are given and constant. Then, we obtain the relation represented by the transfer function $H_{C A V}(s)$

$$
\left[\begin{array}{l}
y_{1}^{c}(s) \\
y_{2}^{c}(s)
\end{array}\right]=H_{C A V}(s)\left[\begin{array}{l}
u_{1}^{c}(s) \\
u_{2}^{c}(s)
\end{array}\right]
$$

Note that $H_{C A V}(s)$ has no zeros at the origin in the complex plane. Let $\bar{y}_{1}^{c}, \bar{y}_{2}^{c}$ be the steady state value of $y_{1}^{c}, y_{2}^{c}$, respectively and let $\bar{u}_{1}^{c}, \bar{u}_{2}^{c}$ be the steady state value of $u_{1}^{c}, u_{2}^{c}$, respectively. Then,

$$
\left[\begin{array}{l}
\bar{y}_{1}^{c} \\
\bar{y}_{2}^{c}
\end{array}\right]=H_{C A V}(0)\left[\begin{array}{l}
\bar{u}_{1}^{c} \\
\bar{u}_{2}^{c}
\end{array}\right] \text {. }
$$

$H_{C A V}(0)$ can be obtained by applying any steady state value test[1],[6]. One method is step input test [6]. Select $u_{1}^{c 1}$ as nonzero constant, $u_{2}^{c 1}$ as zero, and obtain $y_{1}^{c 1}$ and $y_{2}^{c 1}$. Next, select $u_{1}^{c 2}$ as zero, $u_{2}^{c 2}$ as nonzero constant and obtain $y_{1}^{c 2}$ and $y_{2}^{c 2}$. Then, $H_{C A V}(0)$ satisfies

$$
\left[\begin{array}{ll}
y_{1}^{c 1} & y_{1}^{c 2} \\
y_{2}^{c 1} & y_{2}^{c 2}
\end{array}\right]=H_{C A V}(0)\left[\begin{array}{cc}
u_{1}^{c 1} & u_{1}^{c 2} \\
u_{2}^{c 1} & u_{2}^{c 2}
\end{array}\right]
$$

Since Cavity has no zeros at the origin in the complex plane,

$$
\left[\begin{array}{l}
\bar{u}_{1}^{c} \\
\bar{u}_{2}^{c}
\end{array}\right]=H_{C A V}^{-1}(0)\left[\begin{array}{l}
\bar{y}_{1}^{c} \\
\bar{y}_{2}^{c}
\end{array}\right] \text {. }
$$

Note that inputs $u_{1}^{c}$ and $u_{2}^{c}$ of Cavity are given by equations of the normalized amplitude $y_{1}^{k}$ and the normalized phase $y_{2}^{k}$.

$$
\left[\begin{array}{l}
u_{1}^{c} \\
u_{2}^{c}
\end{array}\right]=\left[\begin{array}{l}
10 \sqrt{K P m} \cdot y_{1}^{k} \cdot \cos \left(y_{2}^{k}\right) \\
10 \sqrt{K P m} \cdot y_{1}^{k} \cdot \sin \left(y_{2}^{k}\right)
\end{array}\right]
$$


Since Cavity has no zeros at the origin in the complex plane,

$$
\left[\begin{array}{c}
10 \sqrt{K P m} \cdot y_{1}^{k} \cdot \cos \left(y_{2}^{k}\right) \\
10 \sqrt{K P m} \cdot y_{1}^{k} \cdot \sin \left(y_{2}^{k}\right)
\end{array}\right]=H_{C A V}^{-1}(s)\left[\begin{array}{c}
y_{1}^{c} \\
y_{2}^{c}
\end{array}\right]
$$

Let $\bar{y}_{1}^{k}, \bar{y}_{2}^{k}$ be the steady state values of $y_{1}^{k}, y_{2}^{k}$, respectively and let $\bar{y}_{1}^{c}, \bar{y}_{2}^{c}$ be the steady state values of $y_{1}^{c}, y_{1}^{c}$, respectively. Then, the steady state relation is given by

$$
\left[\begin{array}{c}
\bar{y}_{1}^{k} \cdot \cos \left(\bar{y}_{2}^{k}\right) \\
\bar{y}_{1}^{k} \cdot \sin \left(\bar{y}_{2}^{k}\right)
\end{array}\right]=\frac{1}{10 \sqrt{K P m}} H_{C A V}^{-1}(0)\left[\begin{array}{c}
\bar{y}_{1}^{c} \\
\bar{y}_{2}^{c}
\end{array}\right] \text {. }
$$

Setting

$$
\left[\begin{array}{c}
\bar{y}_{1}^{c} \\
\bar{y}_{2}^{c}
\end{array}\right]=\left[\begin{array}{l}
y_{1 d}^{c} \\
y_{2 d}^{c}
\end{array}\right]
$$

and plugging (56) into (65), we obtain

$$
\left[\begin{array}{c}
\bar{y}_{1}^{k} \cdot \cos \left(\bar{y}_{2}^{k}\right) \\
\bar{y}_{1}^{k} \cdot \sin \left(\bar{y}_{2}^{k}\right)
\end{array}\right]=\frac{1}{10 \sqrt{K P m}} H_{C A V}^{-1}(0)\left[\begin{array}{rr}
F A \cdot \cos (G D) & -F A \cdot \sin (G D) \\
F A \cdot \sin (G D) & F A \cdot \cos (G D)
\end{array}\right]^{-1}\left[\begin{array}{c}
F L D I_{d} \\
F L D \_Q_{d}
\end{array}\right] .
$$

Define the right-hand side of the above equation to be

$$
\left[\begin{array}{c}
\psi_{1} \\
\psi_{2}
\end{array}\right]=\frac{1}{10 \sqrt{K P m}} H_{C A V}^{-1}(0)\left[\begin{array}{rr}
F A \cdot \cos (G D) & -F A \cdot \sin (G D) \\
F A \cdot \sin (G D) & F A \cdot \cos (G D)
\end{array}\right]^{-1}\left[\begin{array}{l}
F L D I_{d} \\
F L D Q_{d}
\end{array}\right]
$$

Then,

$$
\begin{aligned}
& \bar{y}_{1}^{k}=\sqrt{\psi_{1}^{2}+\psi_{2}^{2}} \\
& \bar{y}_{2}^{k}=\tan ^{-1}\left(\frac{\psi_{2}}{\psi_{1}}\right)
\end{aligned}
$$

where $\bar{y}_{1}^{k}$ and $\bar{y}_{2}^{k}$ are the normalized amplitude and the normalized phase of Klystron which yield the desired value FLD_I $I_{d}$ of FLD_I and the desired value FLD_Q ${ }_{d}$ of FLD_Q.

The steady state values $\bar{z}_{1 s}, \bar{z}_{2 s}$, which are the desired values of $\bar{z}_{1}, \bar{z}_{2}$, respectively are obtained by solving the algebraic equations generated from $(41),(42),(68)$ and (69).

$$
\begin{aligned}
& \sum_{i=1}^{N} c_{i} e^{-f_{i} M \bar{z}_{1 s}}=\bar{y}_{1}^{k} \\
& \sum_{i=1}^{N} d_{i} e^{-f_{i} M \bar{z}_{1 s}}+\bar{z}_{2 s}=\bar{y}_{2}^{k} .
\end{aligned}
$$


We have to solve $\bar{z}_{1 s}$ and $\bar{z}_{2 s}$. Instead of obtaining the analytic solution of the system (70) and (71), we obtain the numerical solution by resorting to an optimization method. Our approach is the minimization in the least square sense given as follow:

$$
\operatorname{minimize}\left(\sum_{i=1}^{N} c_{i} e^{-f_{i} M \bar{z}_{1 s}}-\bar{y}_{1}^{k}\right)^{2}+\left(\sum_{i=1}^{N} d_{i} e^{-f_{i} M \bar{z}_{1 s}}+\bar{z}_{2 s}-\bar{y}_{2}^{k}\right)^{2}
$$

The controller design procedure is as follows:

\section{Controller design procedure}

1. Obtain $H_{C A V}(0)$ by applying a step input test.

2. Given desired FLD_I $I_{d}$ and FLD_Q ${ }_{d}$, obtain $\bar{y}_{1}^{k}$ and $\bar{y}_{2}^{k}$.

3. For the solutions $\bar{y}_{1}^{k}$ and $\bar{y}_{2}^{k}$, solve the optimization problem and obtain $\bar{z}_{1 s}$ and $\bar{z}_{2 s}$.

4. Obtain $k_{1}$ and $k_{2}$ so that the matrix $\bar{A}_{z c}$ is stable.

5. Find the solution $R_{z}$ of $0=\bar{A}_{z c} \bar{z}+R_{z}$.

6. Obtain the control input $\bar{u}$ as given in (51) and the control input $u_{z}$ as given in (49).

\section{Numerical Simulation}

We consider the Klystron RF Cavity system when there is $20,000 \mathrm{~Hz}$ sinusoidal ripple and $720 \mathrm{~Hz}, 120 \mathrm{~Hz}$ ripples as well. The maximum power $\mathrm{KP}_{m}$ and the klystron gain $\mathrm{K}_{g}$ are given in Table 6.

\begin{tabular}{||c|r||}
\hline \hline$K_{g}$ & 8449.4 \\
\hline$K P_{m}$ & $3.600 \mathrm{e}+006$ \\
\hline
\end{tabular}

Table 6. Klystron gain and klystron maximum power 

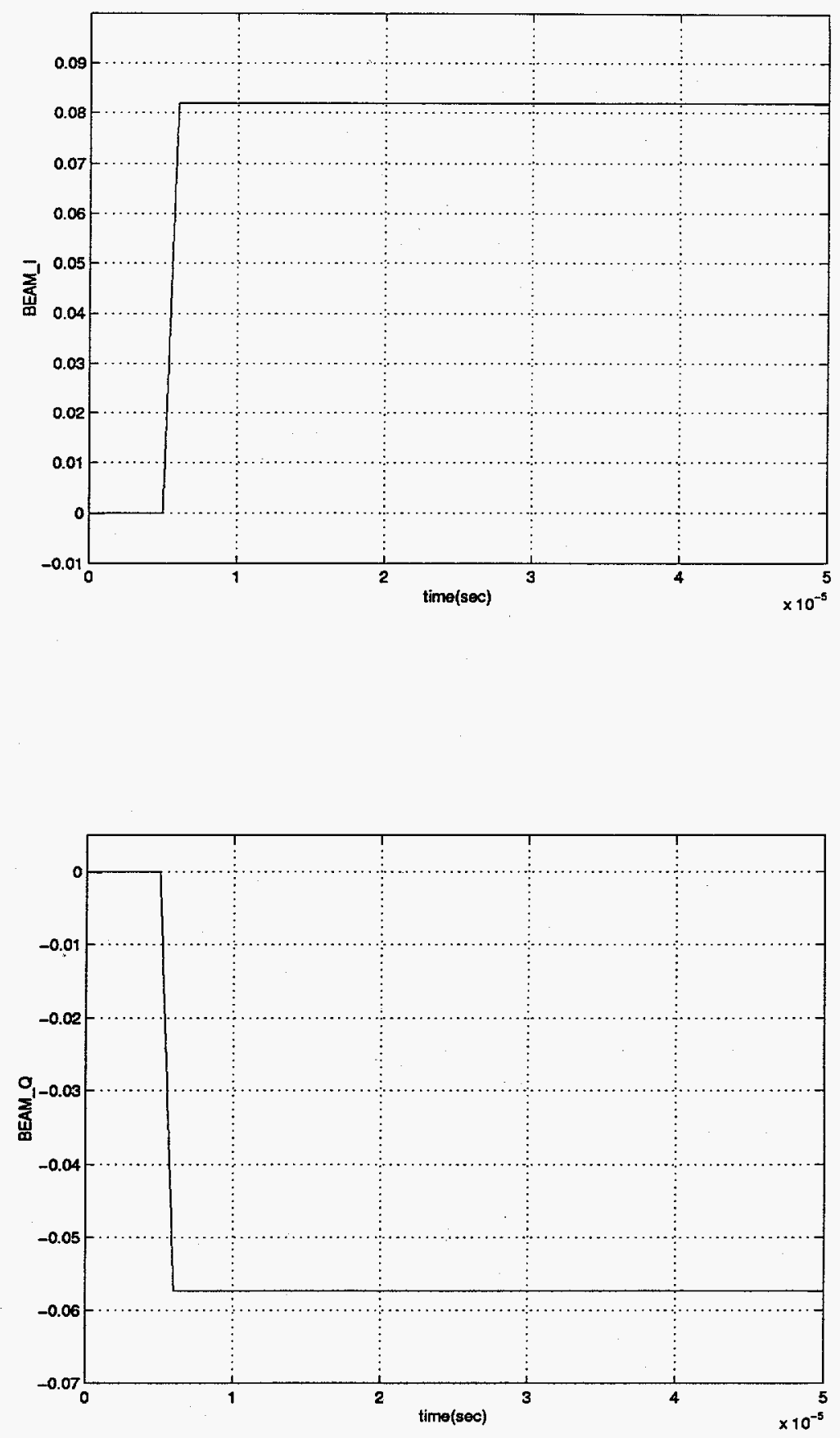

Figure 4: BEAM_I and BEAM_Q used for steady state gain simulation. 
1. For steady state gain $H_{C A V}(0)$, we use the constant input method. BEAM I and BEAM_Q used for the steady state gain simulation is in Figure 4.

First, we set $\bar{u}_{1}^{c}=13226.0$ and $\bar{u}_{2}^{c}=0.0$. We simulated the RF Cavity and obtained the steady state values $\bar{y}_{1}^{c}$ and $\bar{y}_{2}^{c}$. Second, we set $\bar{u}_{1}^{c}=0.0$ and $\bar{u}_{2}^{c}=13226.0$. We simulated the RF Cavity and obtained the steady state values $\bar{y}_{1}^{c}$ and $\bar{y}_{2}^{c}$. Table 7 shows the results.

\begin{tabular}{||c||c|c||c|c||}
\hline \hline Simulation No. & $\bar{u}_{1}^{c}$ & $\bar{u}_{2}^{c}$ & $\bar{y}_{1}^{c}$ & $\bar{y}_{2}^{c}$ \\
\hline \hline 1 & 13226.0 & 0.0 & $1.32243 \mathrm{e}+004$ & $-1.75200 \mathrm{e}+000$ \\
\hline 2 & 0.0 & 13226.0 & $-1.91973 \mathrm{e}+002$ & $1.74330 \mathrm{e}+004$ \\
\hline \hline
\end{tabular}

Table 7. Steady state gain simulation

From the simulation results as given in Table 7 , we compute the steady state gain $H_{C A V}(0)$.

$$
H_{C A V}(0)=\left[\begin{array}{rr}
0.99987 & -0.01452 \\
-0.00013 & 1.31809
\end{array}\right] \text {. }
$$

2. Let the desired FLD $I_{d}$ and FLD_Q $Q_{d}$ be FLD $I_{d}=5.0$ and FLD_Q ${ }_{d}=0.0$. From (67), (68), and (69), we obtain $\bar{y}_{1}^{k}$ and $\bar{y}_{2}^{k}$ as given in Table 8 .

\begin{tabular}{||l|l||}
\hline \hline $\bar{y}_{1}^{k}$ & $6.97080 \mathrm{e}-001$ \\
\hline $\bar{y}_{2}^{k}$ & $6.22858 \mathrm{e}-004$ \\
\hline \hline
\end{tabular}

Table 8. Steady state normalized Amplitude $\bar{y}_{1}^{k}$ and the normalized phase $\bar{y}_{2}^{k}$

3. In order to solve the optimization problem (72), we made use of the unconstrained optimization algorithm in Matlab Toolbox. With initial values $\bar{z}_{1 s}=1.0$ and $\bar{z}_{2 s}=1.0$, after 54 iterations, we obtain the optimal solution $\bar{z}_{1 s}$ and $\bar{z}_{2 s}$ as given in Table 9. 


\begin{tabular}{||l|l||}
\hline \hline $\bar{z}_{1 s}$ & 1.20825 \\
\hline $\bar{z}_{2 s}$ & 0.01088 \\
\hline \hline
\end{tabular}

Table 9. The solution $\bar{z}_{1 s}$ and $\bar{z}_{2 s}$ of the optimization problem (72)

4. Since $a_{1}^{\prime}=2.82486 e+006$ is sufficiently large, we set $k_{1}=-2.0 a_{1}$. And we set $k_{2}=-10 a_{1}$. Hence, the eigenvalues of $\bar{A}_{z c}$ are $\lambda_{1}=-3 a_{1}$ and $\lambda_{2}=-10 a_{1}$ for any $R(t)$ and $\dot{R}(t)$.

5. The solution $R_{z}$ of (54) is given by

$$
R_{z}=\left[\begin{array}{l}
3.41312 e+006 \\
3.07291 e+005
\end{array}\right]
$$

Based on numerical values obtained, we implement the controller (50) to drive the Klystron-RF Cavity system.

Figure 5 through Figure 7 show the simulation results of the Klystron-RF Cavity system in Matlab/Simulink environment. 

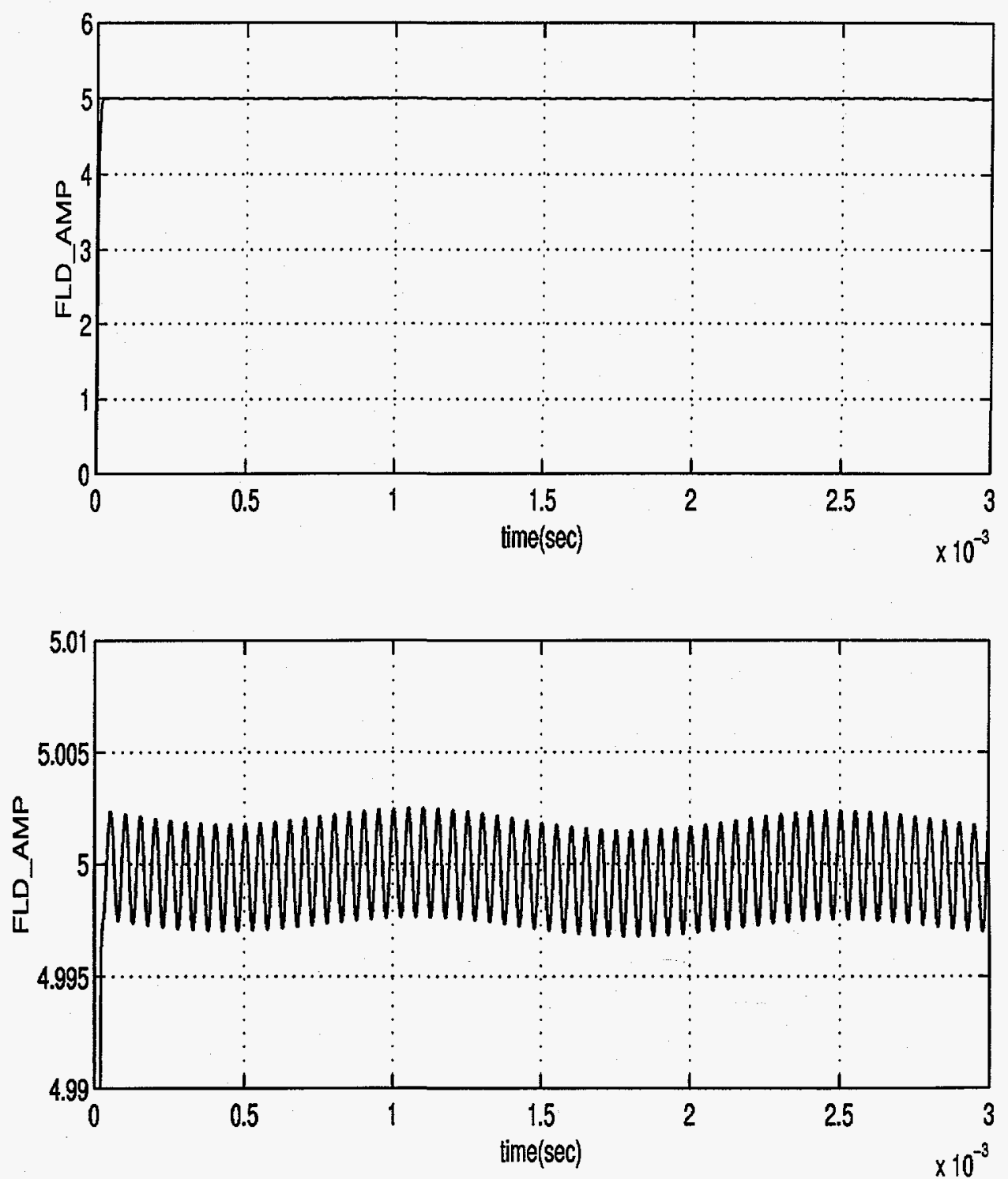

Figure 5: Field Amplitude, FLD_AMP 

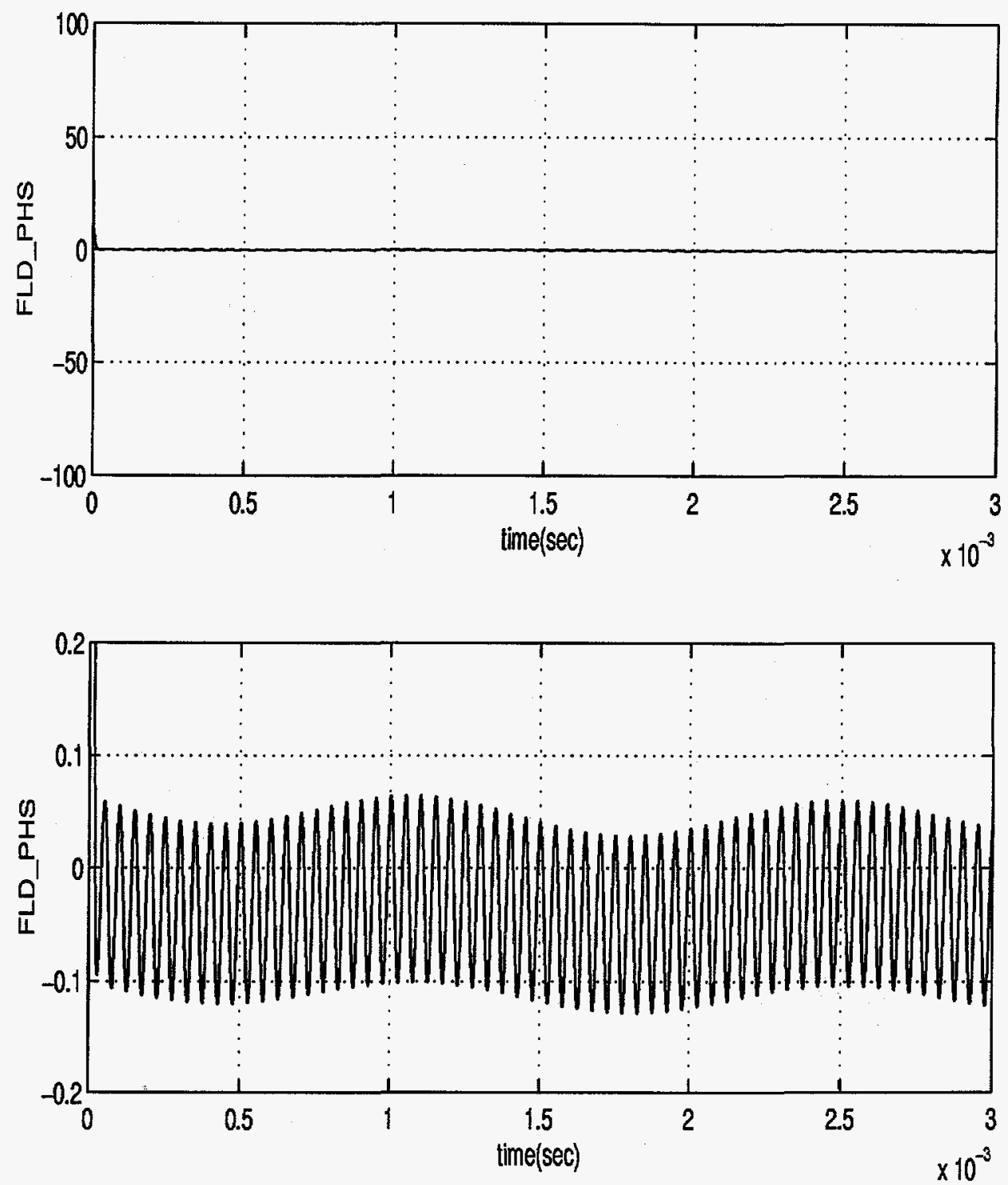

Figure 6: Field Phase, FLD_PHS 

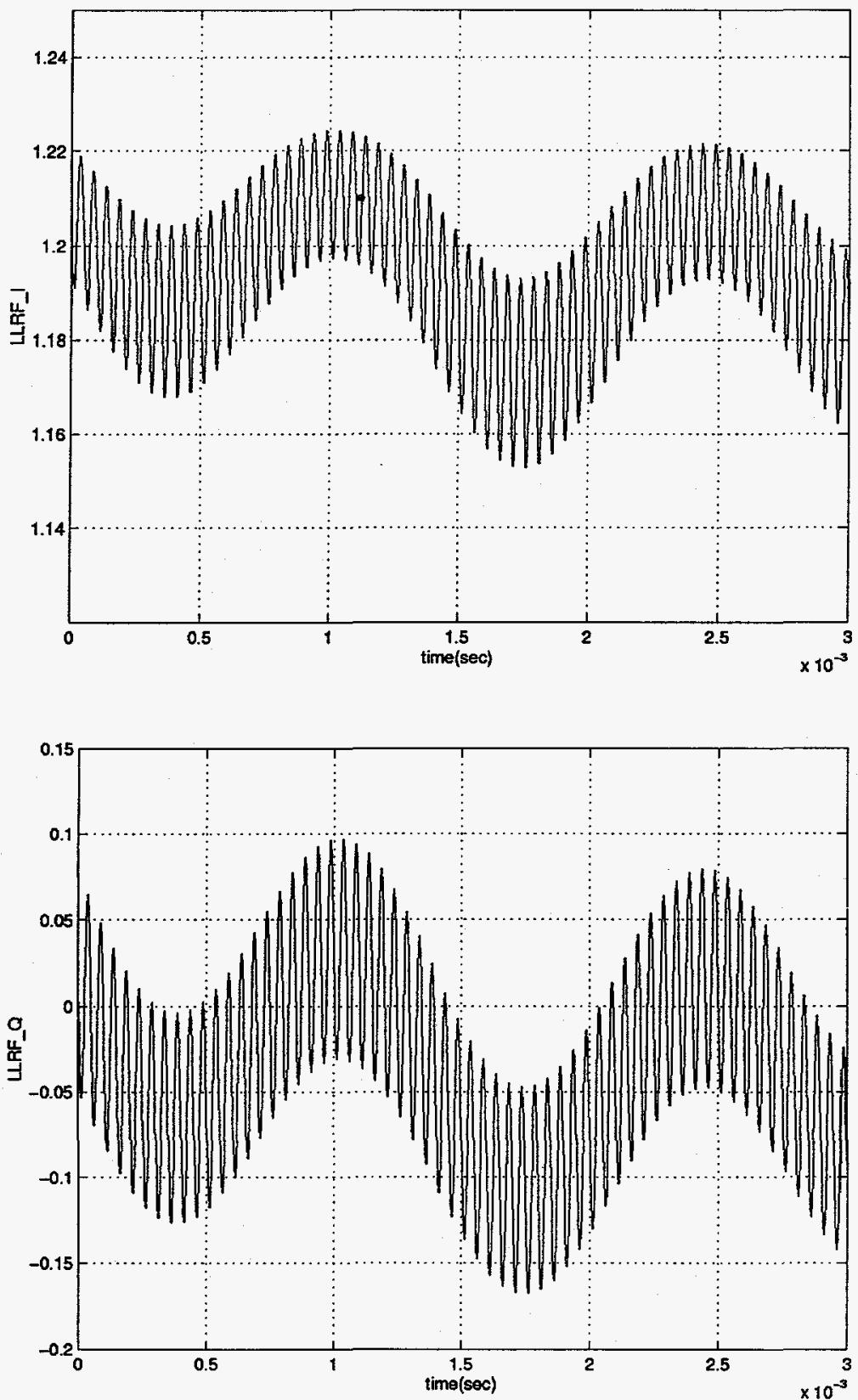

Figure 7: Control input LLRF_I and LLRF_Q 


\section{RIPPLE Estimation}

The controller proposed in the previous section is based on the assumption that the information on ripple $R(t)$ and its time derivative $\frac{d R(t)}{d t}$ are fully known. When a designer has poor information on $R(t)$ and $\frac{d R(t)}{d t}$, the feedback linearization cannot give desired performance. Table 10 and Table 11 show amplitude and phase errors when there are phase difference and amplitude difference between the ripple used for controller and the real ripple entered a klystron. Table 10 is the results when there is a phase difference between the ripple of the feedback linearization controller and the ripple of the klystron. The controller ripple is $R(t)=\sin (2 \pi f t)$ and the ripple of the klystron is $R(t)=\sin (2 \pi f t+\phi), f=120 K H z$. Here, $\phi$ is the phase of the ripple of klystron.

\begin{tabular}{||c|c|c|}
\hline \hline $\begin{array}{c}\text { Ripple phase } \\
\phi(\text { rad })\end{array}$ & $\begin{array}{c}\text { peak-to-peak } \\
\text { field Phase error(Degrees) }\end{array}$ & $\begin{array}{c}\text { peak-to-peak } \\
\text { field Amplitude error(Volts) }\end{array}$ \\
\hline \hline 0.0 & 0.00610 & $0.00006(0.00115 \%)$ \\
\hline$\frac{\pi}{8}$ & 1.12830 & $0.02368(0.47 \%)$ \\
\hline$\frac{2 \pi}{8}$ & 2.21690 & $0.04000(0.80 \%)$ \\
\hline$\frac{4 \pi}{8}$ & 4.10113 & $0.08600(1.72 \%)$ \\
\hline \hline
\end{tabular}

Table 10. Amplitude error and Phase error when there is difference in phase of ripple $R(t)$

Table 11 is the results when there is amplitude difference between the ripple of the feedback linearization controller and the ripple of the klystron. The controller ripple is $R(t)=$ $A_{R} \sin (2 \pi f t), A_{R}=1.0, f=120 K H z$ and the ripple of the klystron is $R(t)=A_{k} \sin (2 \pi f t)$, $f=120 \mathrm{~K} \mathrm{~Hz}$. Here, $A_{k}$ is the amplitude of the ripple of klystron.

\begin{tabular}{||c|c|c|}
\hline \hline $\begin{array}{c}\text { Klystron Ripple } \\
\text { amplitude } A_{k}\end{array}$ & $\begin{array}{c}\text { peak-to-peak } \\
\text { field Phase error(Degrees) }\end{array}$ & $\begin{array}{c}\text { peak-to-peak } \\
\text { field Amplitude error(Volts) }\end{array}$ \\
\hline \hline 0.6 & 1.16286 & $0.02432(0.49 \%)$ \\
\hline 0.8 & 0.58323 & $0.01219(0.24 \%)$ \\
\hline 1.2 & 0.57660 & $0.01209(0.24 \%)$ \\
\hline 1.4 & 1.15631 & $0.02423(0.49 \%)$ \\
\hline \hline
\end{tabular}

Table 11. Amplitude error and Phase error when there is difference in amplitude of ripple $R(t)$ 
The purpose of the low level RF control(LLRF) system is to maintain the field stability within $\pm 1.0 \%$ amplitude and $1.0^{\circ}$ phase. In the case that there is phase difference, only $\frac{\pi}{8}$ phase difference yields $1.12^{\circ}$ field phase error. In the case that there is amplitude difference, $40 \%$ gap of amplitude yields $1.156^{\circ}$ field phase error.

For the remedy to the poor information on the ripple and its time derivative, we can make use of Lyapunov redesign after we design the exact feedback linearization controller based on the nominal values of the ripple and its time derivative[4]. This additional controller compensates the uncertainties or unmodelled dynamics. Another possible remedy is to design the estimator which yields the estimated ripple and its time derivative and based on the estimated information, we design the controller.

In this section, we address the ripple estimator which estimates the ripple $R(t)$ and its time derivative $\frac{d R(t)}{d t}$, and the feedback linearization controller based on the estimator.

We first consider equations as given in (21) and (22).

$$
\begin{aligned}
& z_{1}=\sqrt{x_{1}^{2}+x_{2}^{2}} \\
& z_{2}=\tan ^{-1}\left(\frac{x_{2}}{x_{1}}\right)
\end{aligned}
$$

where $x_{1}$ and $x_{2}$ satisfy

$$
\begin{aligned}
& \dot{x}_{1}=-a_{1} x_{1}+a_{1} u_{1} \\
& \dot{x}_{2}=-a_{1} x_{2}+a_{1} u_{2}
\end{aligned}
$$

and $u_{1}=$ LLRF $I, u_{2}=$ LLRF_Q. Given LLRF $\perp$ and LLRF_Q , we can obtain $z_{1}$ and $z_{2}$ by solving differential equations (75), (76) and algebraic equations (73), (74).

Second, we consider equations given by (19) and (20)

$$
\begin{aligned}
& H P R F \_I=10 \sqrt{K P_{m}} \cdot y_{1}^{k} \cdot \cos \left(y_{2}^{k}\right) \\
& H P R F_{-} Q=10 \sqrt{K P_{m}} \cdot y_{1}^{k} \cdot \sin \left(y_{2}^{k}\right) .
\end{aligned}
$$

From (77) and (78), for given HPRF_I and HPRF_Q, we obtain the normalized amplitude $y_{1}^{k}$ and the normalized phase $y_{2}^{k}$ of the klystron by solving algebraic equations.

$$
\begin{aligned}
y_{1}^{k} & =\frac{1}{10 \sqrt{K P_{m}}} \sqrt{H P R F_{-} I^{2}+H P R F_{-} Q^{2}} \\
y_{2}^{k} & =\tan ^{-1}\left(\frac{H P R F_{-} Q}{H P R F_{-} I}\right) .
\end{aligned}
$$

Third, we consider the klystron model as given in Figure 1. In Figure 1, the normalized amplitude of the klystron is the output of the look-up table AMPLITUDE SATURA- 
TION and the input of the look-up table AMPLITUDE SATURATION is given by

$$
A=\frac{K_{g}}{10 \sqrt{K P_{m}}}(0.01 R(t)+1)^{1.25} \cdot z_{1}
$$

in $z$-coordinate, or

$$
A=\frac{K_{g}}{10 \sqrt{K P_{m}}} \bar{z}_{1}
$$

in $\bar{z}$-coordinate. Also, there exists a region of $\left(A, y_{1}^{k}\right)$ pairs where there is an inverse look-up table of the look-up table AMPLITUDE SATURATION. This region can be extracted from data given in Table 1 and Table 2. As in the case of AMPLITUDE SATURATION, we obtain the curve fitting equation for the inverse look-up table for AMPLITUDE SATURATION. Since in the controller design, we make use of the output equations (35) and (36) or (41) and (42) which are based on the curve fitting equation, we use the output equation as given in (41) in order to obtain the curve fitting equation for the inverse look-up table for AMPLITUDE SATURATION within the region of invertibility. Based on the generated data pairs from (41) where the selected data of $y_{1}^{k}$ and $\bar{z}_{1}$ guarantee invertibility, we obtain the curve fitting equation as follows.

$$
\bar{z}_{1}=\sum_{i=1}^{N} c_{i}^{z} e^{-f_{i}^{z} y_{1}^{k}}
$$

where $N=7$, coefficients $f_{i}^{z}, i=1,2, \cdots, N$ and the coefficients $c_{i}^{z}, i=1,2, \cdots, N$ obtained are given in the Table 12 .

\begin{tabular}{||c|c||c|r||}
\hline \hline$f_{1}^{z}$ & 0.50 & $c_{1}^{z}$ & 246379.701273592 \\
\hline$f_{2}^{z}$ & 0.75 & $c_{2}^{z}$ & -1633291.85956396 \\
\hline$f_{3}^{z}$ & 1.00 & $c_{3}^{z}$ & 4505197.57531207 \\
\hline$f_{4}^{z}$ & 1.25 & $c_{4}^{z}$ & -6618176.95439792 \\
\hline$f_{5}^{z}$ & 1.50 & $c_{5}^{z}$ & 5460679.73050349 \\
\hline$f_{6}^{z}$ & 1.75 & $c_{6}^{z}$ & -2399431.11098975 \\
\hline$f_{7}^{z}$ & 2.00 & $c_{7}^{z}$ & 438643.015066461 \\
\hline \hline
\end{tabular}

Table 12. Coefficients of Curve fitting equation for Inverse AMPLITUDE SATURATION 
The estimate of the ripple $R(t)$ and the estimate of the time derivative $\frac{d R(t)}{d t}$ of the ripple $R(t)$ are obtained by considering the klystron system both in $z$-coordinate and $\bar{z}$-coordinate. The relationship between $z$-coordinate and $\bar{z}$-coordinate is given by

$$
\begin{aligned}
& \bar{z}_{1}=(0.01 R(t)+1)^{1.25} z_{1} \\
& \bar{z}_{2}=z_{2}+3 \cdot \frac{\pi}{180} \cdot R(t)
\end{aligned}
$$

Whenever $x_{1}$ and $x_{2}$ are obtained from (75) and (76), then we can obtain $z_{1}$ by using (73). Similarly, whenever $y_{1}^{k}$ is obtained from (79), we can obtain $\bar{z}_{1}$ by using (83). For given $z_{1}$ and $\bar{z}_{1}$, we can obtain the estimate $\hat{R}(t)$ of the ripple $R(t)$ by solving algebraic equation (84).

$$
\hat{R}(t)=100\left(\left(\frac{\bar{z}_{1}(t)}{z_{1}(t)}\right)^{0.8}-1.0\right) .
$$

Also, the estimate $\dot{\hat{R}}(t)$ of time derivative $\dot{R}(t)$ of the ripple $R(t)$ is obtained by differentiation of $\hat{R}(t)$.

The feedback linearization controller based on the estimate $\hat{R}(t)$ and $\dot{\hat{R}}(t)$ is given by

$$
\begin{aligned}
& u_{z}=\hat{\bar{B}}_{z}^{-1}(\bar{z}, \hat{R}(t), \dot{\hat{R}}(t))\left(\bar{u}-E_{z}(\hat{R}(t), \dot{\hat{R}}(t))\right) \\
& \bar{u}=\hat{K}(\hat{R}(t), \dot{\hat{R}}(t)) \bar{z}+R_{z}
\end{aligned}
$$

where

$$
\begin{aligned}
& \hat{\bar{B}}_{z}(\bar{z}, \hat{R}(t), \dot{\hat{R}}(t))=\left[\begin{array}{cc}
b_{z 11}(\bar{z}, \hat{R}(t), \dot{\hat{R}}(t)) & b_{z 12}(\bar{z}, \hat{R}(t), \dot{\hat{R}}(t)) \\
b_{z 21}(\bar{z}, \hat{R}(t), \dot{\hat{R}}(t)) & b_{z 22}(\bar{z}, \hat{R}(t), \dot{\hat{R}}(t))
\end{array}\right], \\
& E_{z}(\hat{R}(t), \dot{\hat{R}}(t))=\left[\begin{array}{c}
0 \\
3 \cdot \frac{\pi}{180} \dot{\hat{R}}(t)
\end{array}\right], \\
& \hat{K}(\hat{R}(t), \dot{\hat{R}}(t))=\left[\begin{array}{cc}
k_{1}+0.0125(0.01 \hat{R}(t)+1)^{-1} \dot{\hat{R}}(t) & 0 \\
0 & k_{2}
\end{array}\right], \\
& R_{z}=\left[\begin{array}{c}
r_{1} \\
r_{2}
\end{array}\right] .
\end{aligned}
$$

Figure 8 shows the feedback linearization system in Matlab/Simulink environment. KLYSTRON is the klystron model, RIPPLE is the equivalent system which generates high voltage power supply ripple. RF_CAVITY is the RF Cavity with Beam. Inputs of the ripple estimator are HPRF_I, HPRF_Q, LLRF_I and LLRF_Q which are measurable. The ripple estimator estimates both the ripple and its time derivative. The time derivative information is used 
in the feedback linearization controller and the usage of the time derivative information improves the closed loop performance[10].

Figures 9-12 show the simulation when there is $20,000 \mathrm{~Hz}$ sinusoidal ripple and $720 \mathrm{~Hz}$, $120 \mathrm{~Hz}$ ripples as well.

Figures 13-16 show the simulation when the ripple is

$$
R(t)=1.0 \sin \left(2 \pi f_{1} t\right)+1.0 \sin \left(2 \pi f_{2} t+\frac{3 \pi}{8}\right)
$$

where $f_{1}=120 k H z, f_{2}=80 k H z$.

\section{References}

[1] K. J. Astrom and T. Hagglund, PID controllers:theory, design, and tuning, Second Edition, Instrument Society of America, Research Triangle Park, NC, 1995.

[2] C.T. Chen, Introduction to linear System Theory, Second edition, Rinehart and Winston, New York, 1986.

[3] A. Isidori, Nonlinear Control Systems, Third Edition, Springer Verlag, New York, 1995.

[4] H.K. Khalil, Nonlinear systems, Macmillan, New York, 1992.

[5] S. Kwon, " $0.3 \%$ high voltage power supply ripple response test," preprint.

[6] Z. J. Palmor, Y. Halevi, and N. Krasney, "Automatic tuning of decentralized PID controllers for TITO processes," Automatica, vol. 31, pp. 1001-1010, 1995.

[7] D. Rees, J. Bradley III, K. Cummings, M. Lynch, A. Regan, T. Rohlev, W. Roybal, Y.M. Wang, "Design and test results of the low energy demonstration accelerator(LEDA) RF systems," presented in XIX International LINAC Conference, Chicago, USA, 1998.

[8] A. Regan and C. Ziomek, "APT LLRF control system model results," presented in XIX International LINAC Conference, Chicago, USA, 1998.

[9] W. J. Rugh, "Analytical Framework for Gain Scheduling," IEEE Control Syst. Mag., vol. 11, no. 1, pp. 79-84, 1991.

[10] N. Sureshbabu and W. J. Rugh, "Output Regulation with Derivative Information," IEEE Trans. Automat. Contr., vol. 40, pp. 1755-1766, 1995. 
[11] C. Ziomek and A. Regan, "Simplification of Matrixx Model: Preliminary LLRF System Design," Technical Note, AOT-5-TN:001, RF Technology Group, AOT Division, Los Alamos National Laboratory, 1996.

[12] C. Ziomek and A. Regan, "Superconducting system model; HVPS ripple model," Technical Note, AOT-5-TN:003, RF Technology Group, AOT Division, Los Alamos National Laboratory, 1996.

[13] C. Ziomek and A. Regan, "Model Multiple Klystrons, Pulsed Beam (SC and NC)," Technical Note, AOT-5-TN:005, RF Technology Group, AOT Division, Los Alamos National Laboratory, 1996. 


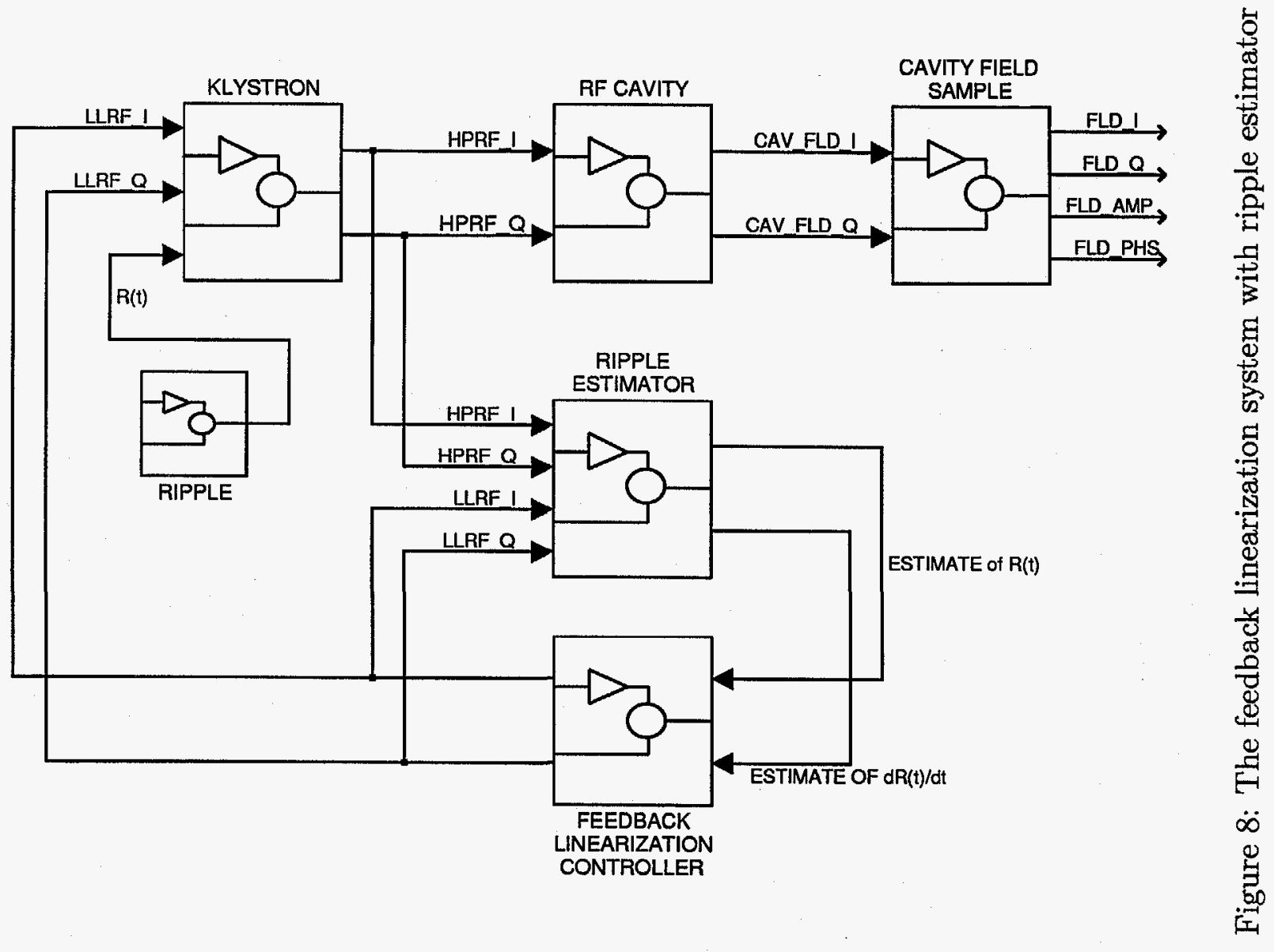



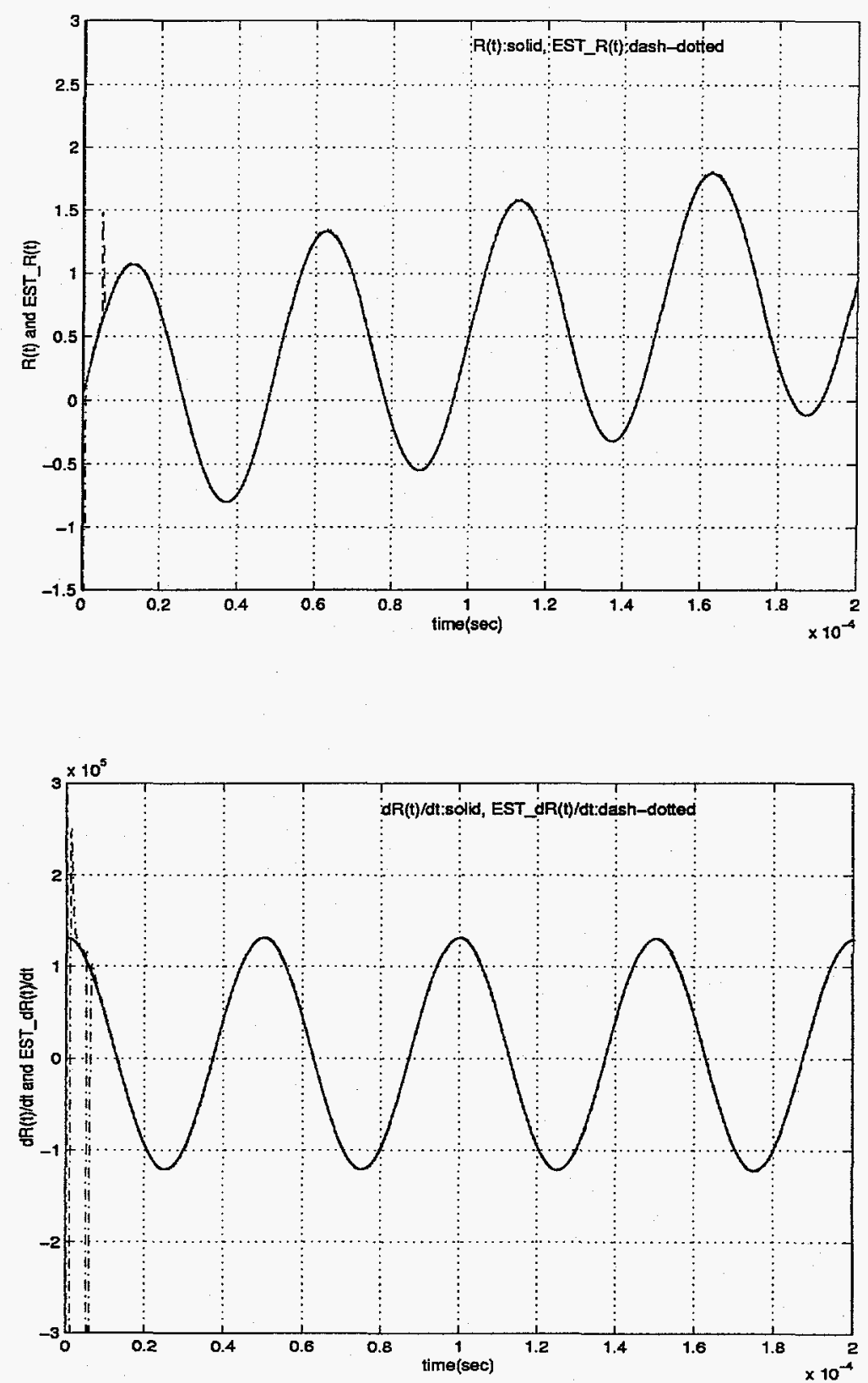

Figure 9: Ripple $R(t)$ and its time derivative $d R(t) / d t$ and their estimates $E S T_{-} R(t)$ and $E S T \_d R(t) / d t$ 

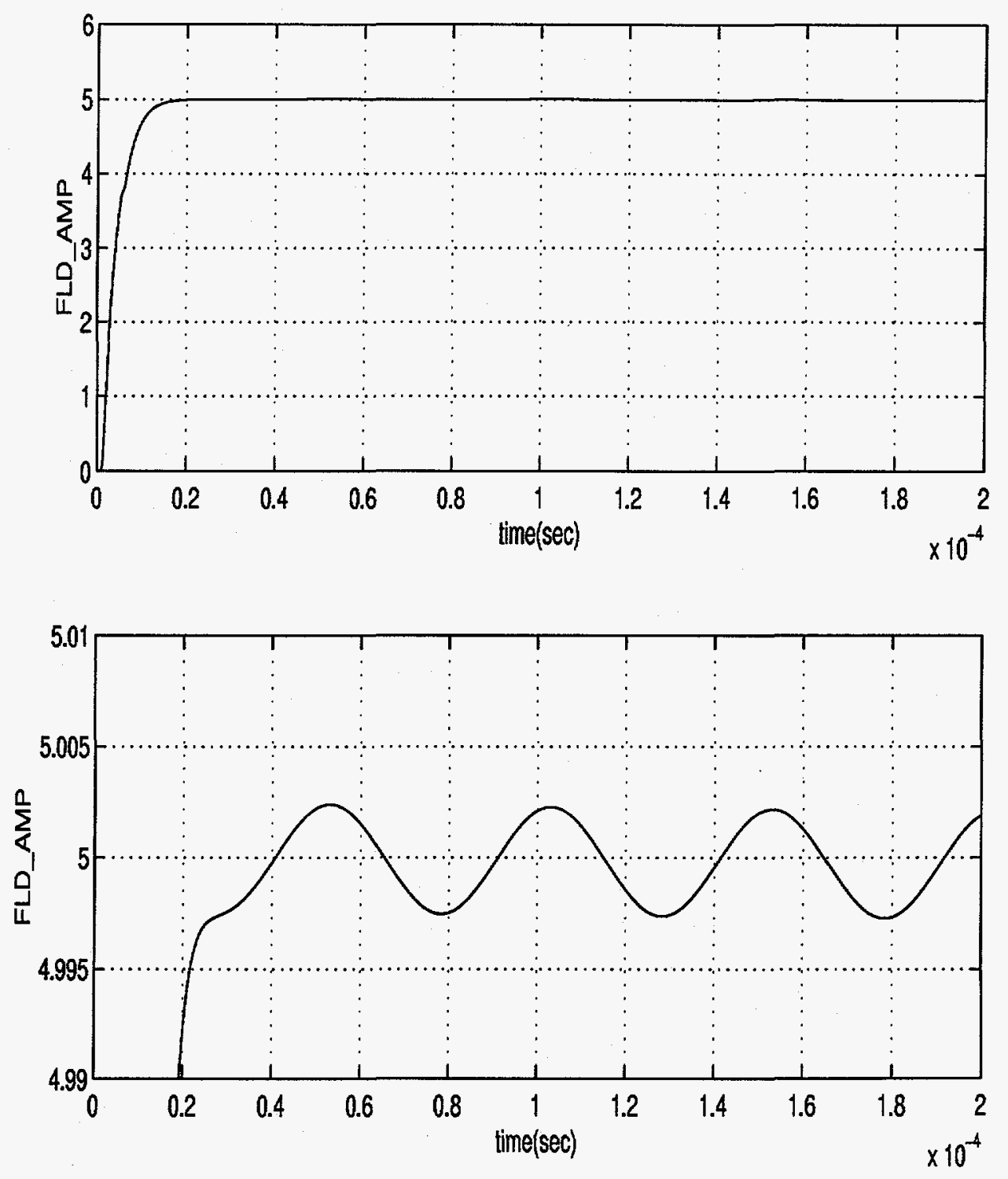

Figure 10: Amplitude of Field, FLD_AMP 

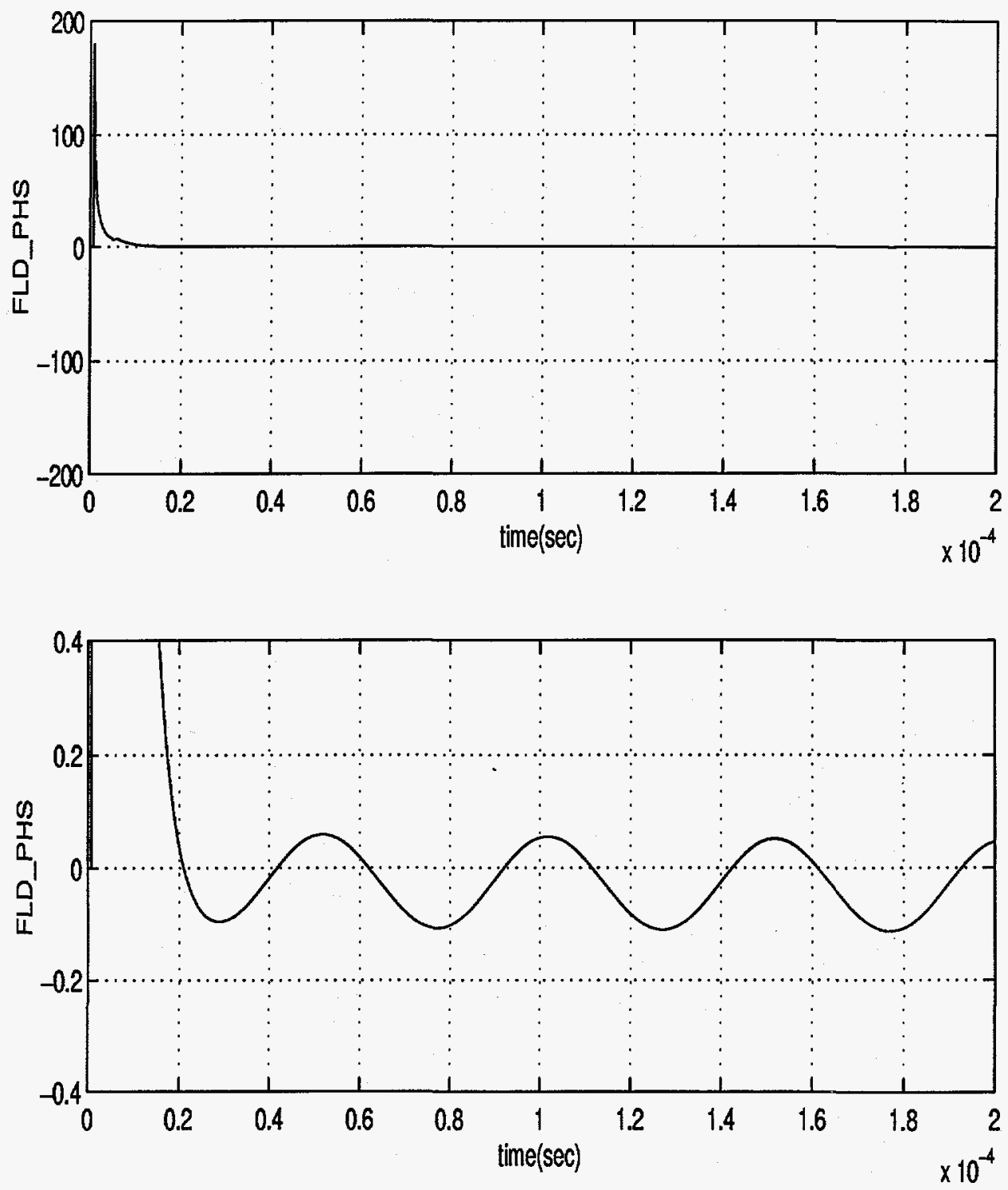

Figure 11: Phase of Field, FLD_PHS 

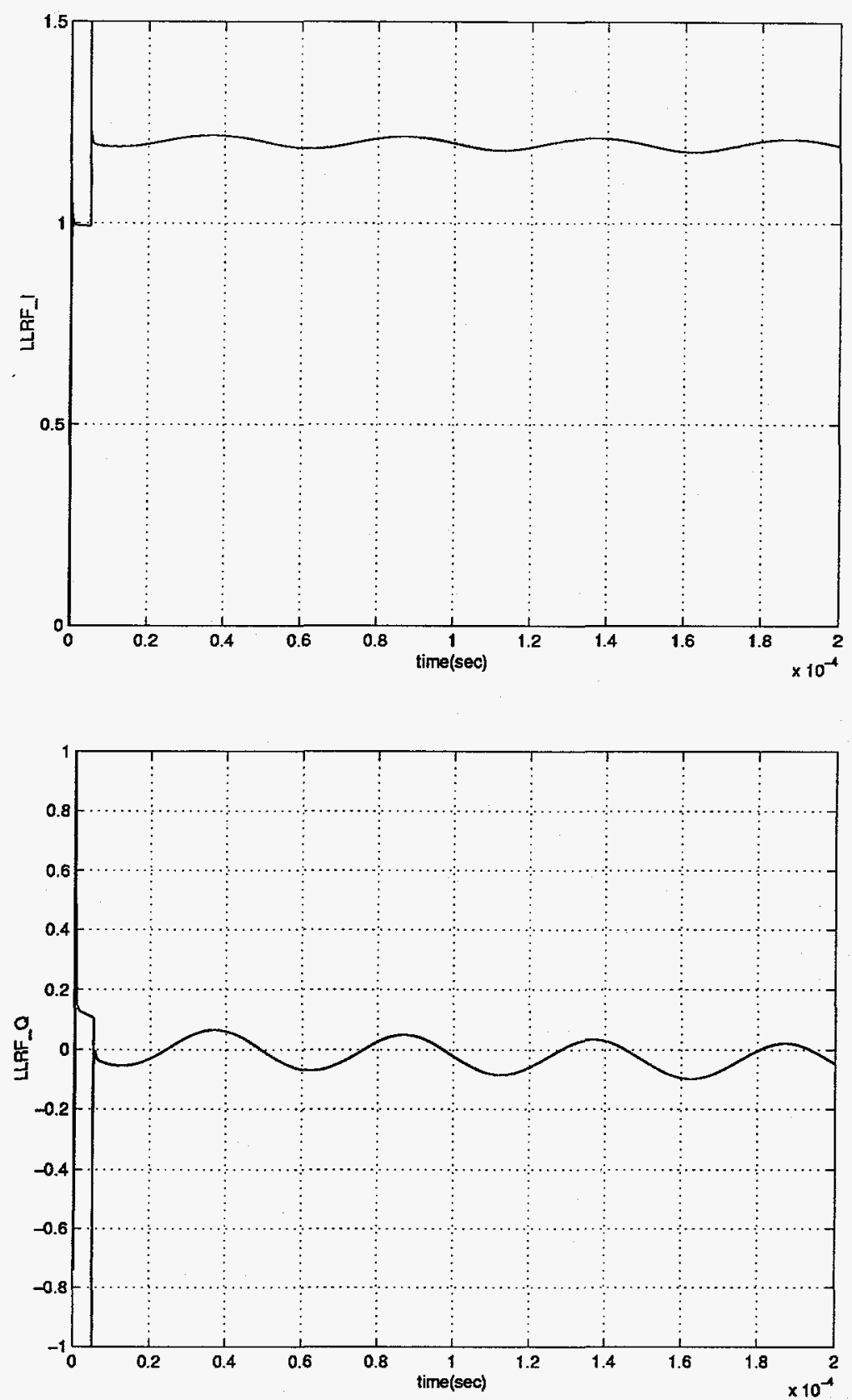

Figure 12: LLRF_I and LLRF_Q 

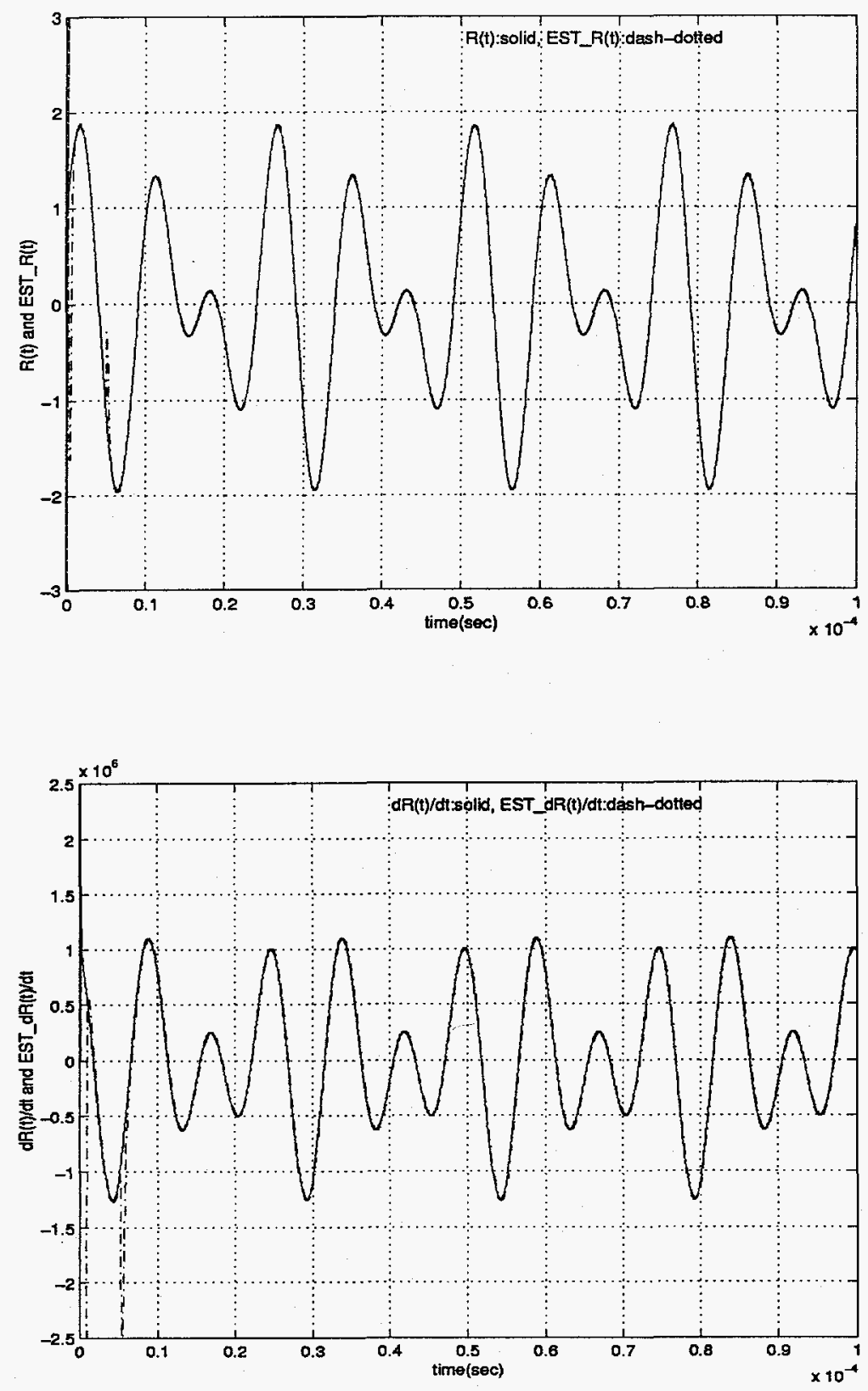

Figure 13: Ripple $R(t)$ and its time derivative $d R(t) / d t$ and their estimates $E S T \_R(t)$ and $E S T_{-} d R(t) / d t$ 

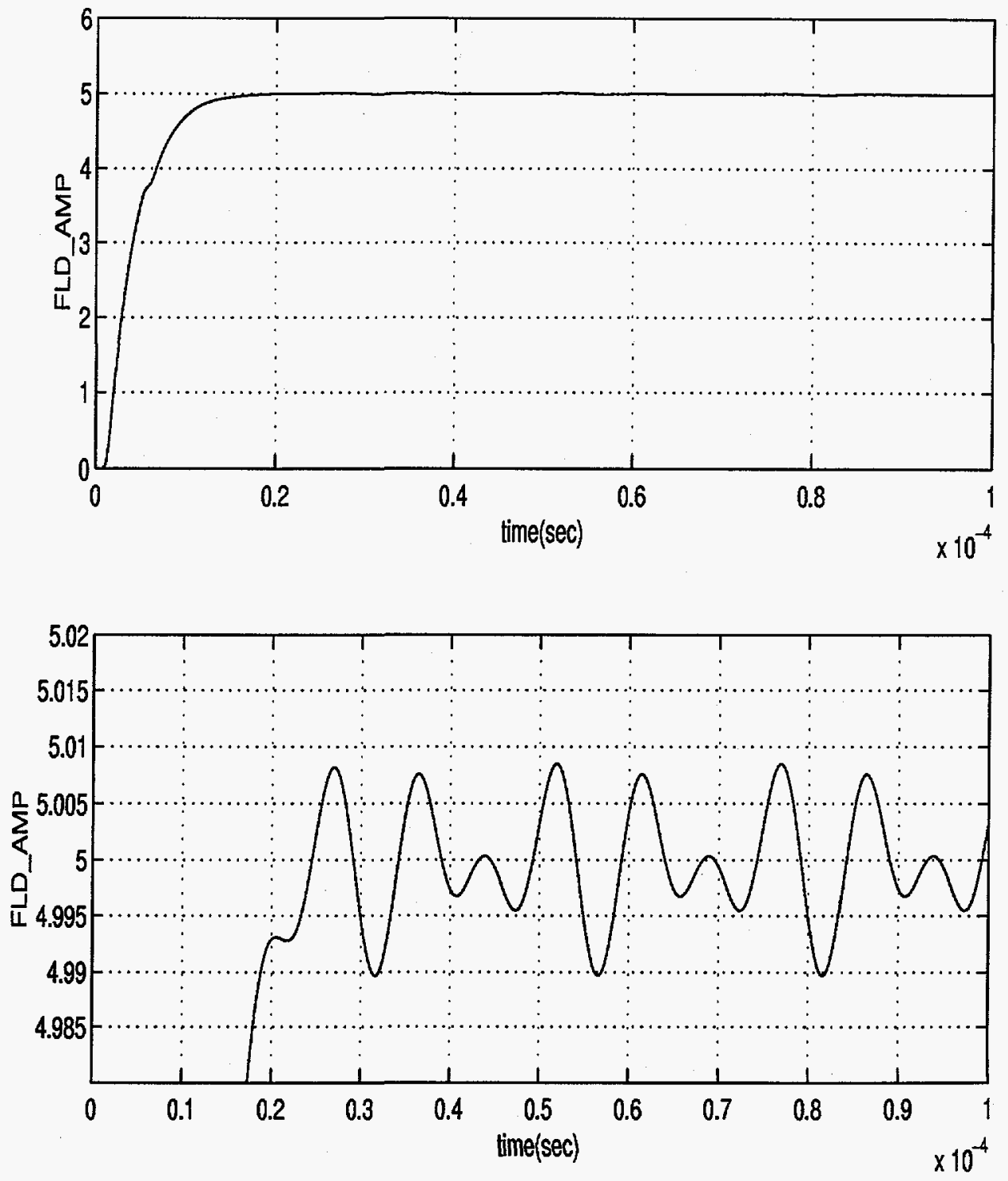

Figure 14: Amplitude of Field, FLD_AMP 

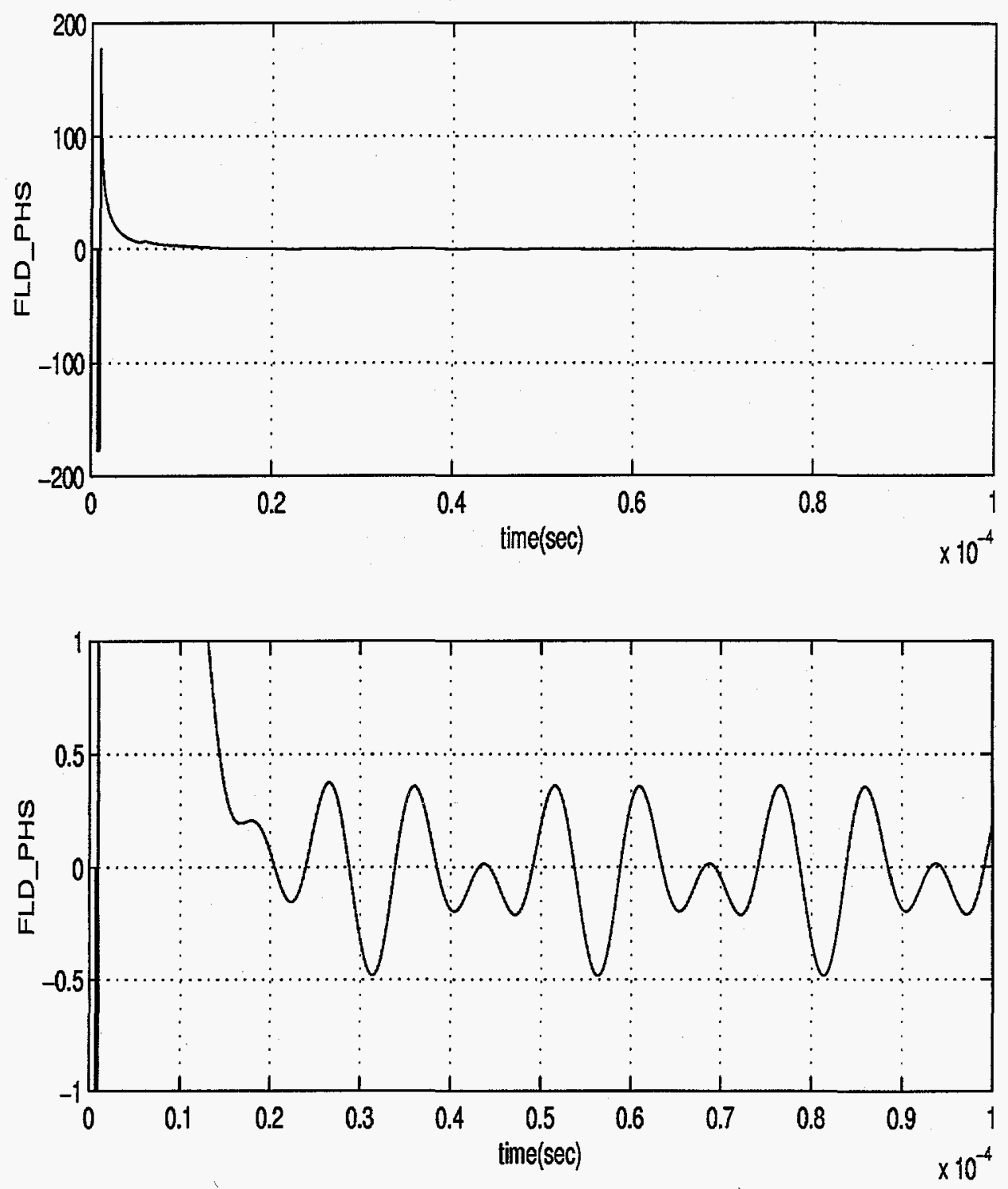

Figure 15: Phase of Field, FLD_PHS 

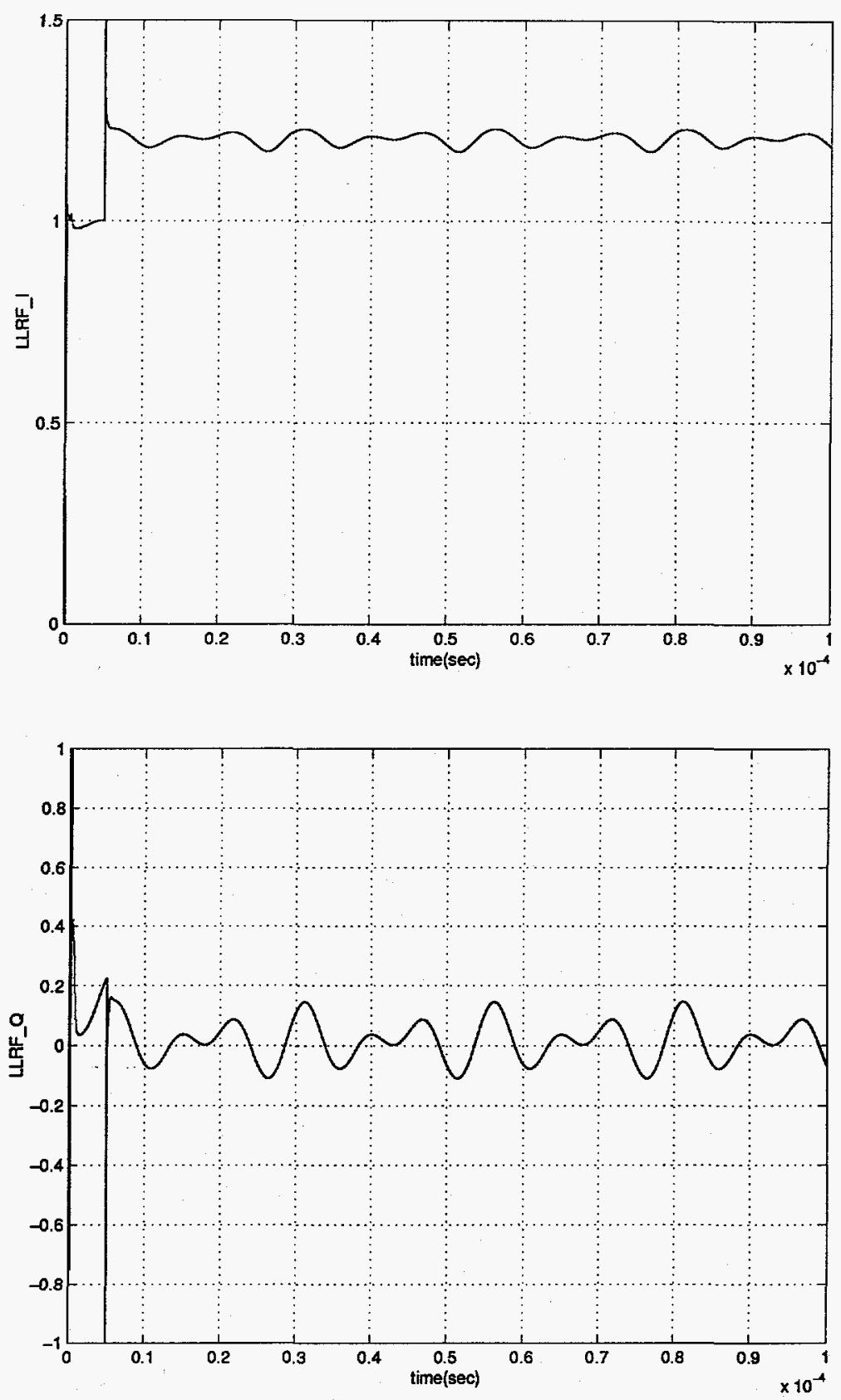

Figure 16: LLRF_I and LLRF_Q 